\title{
Long non-coding RNA gene regulation and trait associations across human tissues
}

Authors: O. M. de Goede ${ }^{1 *}$, N. M. Ferraro ${ }^{2}$, D. C. Nachun ${ }^{3}$, A. S. Rao ${ }^{4}$, F. Aguet $^{5}$, A. N. Barbeira $^{6}$, S. E. Castel ${ }^{7,8}$, S. Kim-Hellmuth ${ }^{7,8,9}$, Y. Park ${ }^{10}$, A. J. Scott ${ }^{11}$, B. J. Strober ${ }^{12}$, GTEx Consortium, C. D. Brown ${ }^{13}$, X. Wen ${ }^{14}$, I. M. Hall ${ }^{11}$, A. Battle ${ }^{12,15}$, T. Lappalainen ${ }^{7,8}$, H. K. Im ${ }^{6}$, K. G. Ardlie ${ }^{5}$, T. Quertermous ${ }^{16}$, K. Kirkegaard ${ }^{1,17}$, S. B. Montgomery ${ }^{1,3 *}$

\section{Affiliations:}

${ }^{1}$ Department of Genetics, Stanford University, Stanford, CA, USA,

${ }^{2}$ Biomedical Informatics Training Program, Stanford University, Stanford, CA, USA,

${ }^{3}$ Department of Pathology, Stanford University, Stanford, CA, USA,

${ }^{4}$ Department of Bioengineering, Stanford University, Stanford, CA, USA,

${ }^{5}$ The Broad Institute of MIT and Harvard, Cambridge, MA, USA,

${ }^{6}$ Section of Genetic Medicine, Department of Medicine, The University of Chicago, Chicago, IL, USA,

${ }^{7}$ New York Genome Center, New York, NY, USA,

${ }^{8}$ Department of Systems Biology, Columbia University, New York, NY, USA,

${ }^{9}$ Statistical Genetics, Max Planck Institute of Psychiatry, Munich, Germany,

${ }^{10}$ Department of Systems Pharmacology and Translational Therapeutics, University of Pennsylvania, Perelman School of Medicine, Philadelphia, PA, USA,

${ }^{11}$ McDonnell Genome Institute, Washington University School of Medicine, St. Louis, MO, USA

12 Department of Biomedical Engineering, Johns Hopkins University, Baltimore, MD, USA,

${ }^{13}$ Department of Genetics, University of Pennsylvania, Perelman School of Medicine, Philadelphia, PA, USA,

${ }^{14}$ Department of Biostatistics, University of Michigan, Ann Arbor, MI, USA

${ }^{15}$ Department of Computer Science, Johns Hopkins University, Baltimore, MD, USA

${ }^{16}$ Division of Cardiovascular Medicine and Cardiovascular Institute, Stanford University, Stanford, CA, USA,

${ }^{17}$ Department of Microbiology and Immunology, Stanford University, Stanford, CA, USA

*Correspondence to: odegoede@ stanford.edu, smontgom@stanford.edu 
Abstract: Long non-coding RNA (lncRNA) genes are known to have diverse impacts on gene regulation. However, it is still a major challenge to distinguish functional lncRNAs from those that are byproducts of surrounding transcriptional activity. To systematically identify hallmarks of biological function, we used the GTEx v8 data to profile the expression, regulation, network relationships and trait associations of lncRNA genes across 49 tissues encompassing 87 distinct traits. In addition to revealing widespread differences in regulatory patterns between IncRNA and protein-coding genes, we identified novel disease-associated lncRNAs, such as C6orf 3 for psoriasis and LINC01475/RP11-129J12.1 for ulcerative colitis. This work provides a comprehensive resource to interrogate lncRNA genes of interest and annotate cell type and human trait relevance.

One Sentence Summary: lncRNA genes have distinctive regulatory patterns and unique trait associations compared to protein-coding genes.

Long non-coding RNA (lncRNA) genes are a prevalent and heterogeneous group of RNA molecules that lack protein-coding potential. They vary in their epigenetic marks, splicing and transcript structure (1-4). Previous studies have demonstrated that lncRNA genes have lower expression, increased tissue-specificity, and greater variability in expression across individuals than protein-coding genes $(1,5-8)$. Despite these differences, many lncRNA genes have been demonstrated to have important roles in gene regulation from epigenetic reprogramming to posttranscriptional regulation $(4,9)$. Although the number of annotated lncRNA genes is increasing as a result of more sensitive transcriptomic profiling in a wide range of contexts $(1,5,10,11)$, it is not known how many of these lncRNAs have important functional consequences.

In this study, we used the Genotype-Tissue Expression (GTEx) project v8 data to profile genetic regulation of lncRNA genes across 49 human tissues. We combine multiple approaches, including expression quantitative trait loci (eQTL) analysis, gene expression outlier analysis, coexpression networks, and colocalization, to identify putative functional lncRNAs, their cellular contexts, and their relevance to human traits.

\section{Literature and database curation produced relevant and comparable sets of IncRNA and protein-coding genes}

lncRNA genes are difficult to study because of their low expression patterns and heterogeneity. To mitigate this challenge, we incorporated three subgroups of genes into our comparisons (Fig. 1A): protein-coding genes that were expression-matched to lncRNA genes (Fig. S1); high-confidence non-coding lncRNA genes, which passed an especially stringent set of criteria to be classified as non-coding (12); and a set of 713 lncRNAs with strong prior evidence of function $(13,14)$ (Table S1) (see Methods). Poly(A) selection was performed prior to RNAsequencing, which can affect the types of lncRNA genes quantified: RNA-sequencing libraries prepared by ribosomal RNA depletion and by poly(A) selection quantify similar numbers of lncRNA genes, but lncRNA genes unique to poly(A) selection tend to be antisense transcripts, 
whereas lncRNA genes unique to ribosomal RNA depletion tend to be intergenic or intronic lncRNA genes (15).

Before comparing patterns of expression and regulation in these gene groups, we first compared the physical properties of these genes. As has been previously described $(6,16)$, protein-coding genes tended to be longer than lncRNA genes (with median transcript lengths of 3,513 and 657 bases, respectively) and have higher numbers of exons (medians of 10 and 2, respectively) (Fig. S2A-C). Lower proportions of lncRNA genes were expressed in each tissue compared to protein-coding genes, and lncRNA isoforms generally had higher transcript support level scores (Fig. S2D-E). Transcript support level scores reflect the quality of primary data supporting the transcript structure, with lower scores indicating a more well-supported transcript model. The higher scores of lncRNA genes are likely related to the relative scarcity of lncRNA transcripts. Many of these physical differences likely informed the more complex regulatory differences we saw throughout these analyses.

\section{IncRNA genes have greater tissue-specificity in gene expression and eQTLs}

The well-established tissue-specificity of lncRNA gene expression $(1,5,6,8,11)$, was apparent across the 49 analyzed tissues. At an expression threshold of TPM $\geq 0.5$ in at least $20 \%$ of samples, most of the genes in both the total lncRNA and the high-confidence non-coding lncRNA gene groups are either not expressed, or only expressed in 1-5 tissues (Fig. 1B). In contrast, the majority of both protein-coding gene groups are expressed in all or nearly all tissues. Expression of lncRNA genes with known function showed intermediate tissuespecificity, with the proportion of genes expressed in only 1-5 tissues significantly lower than total lncRNA genes $\left(\chi^{2}=36.8, \mathrm{p}=1.3 \times 10^{-9}\right.$, test of equal proportions $)$ but significantly greater than total coding genes $\left(\chi^{2}=266.7, \mathrm{p}<2.2 \times 10^{-16}\right.$, test of equal proportions). This may reflect the biases in functional lncRNA identification: namely, genes that are operating in many different contexts are more likely to have their functions discovered.

To explore which tissues had the highest numbers of uniquely expressed genes, we focused on the 28 "broad tissue" categories; these are more general tissue types defined by the GTEx Consortium, to which each of the 49 tissues are assigned. There were 4,114 genes that were broad tissue-specific, that is they were expressed in only one broad tissue type (Table S2). Of these uniquely expressed genes, 3,301 were lncRNA genes and only 813 were protein-coding genes (Fig. 1C). Both groups were dominated by genes with testis-specific expression; this may reflect the "transcriptional scanning" suggested to occur during spermatogenesis to reduce the male germline mutation rate (17). The next highest numbers of broad tissue-specific genes came from the brain, skin, and blood.

In GTEx v8, 94.7\% of protein-coding genes and $67.3 \%$ of lncRNA genes were identified as eGenes (i.e. having at least one eQTL at FDR $\leq 0.05$ ) in at least one tissue (18). Per tissue, approximately $85 \%$ of expressed protein-coding genes and $50 \%$ of expressed lncRNA genes are eGenes (at an expression threshold of TPM $\geq 0.5$ in $>20 \%$ of samples) (Fig. 2A). The lower frequency of lncRNA eGenes may be partly due to their low gene expression, which limited eQTL detection. However, the fact that approximately $75 \%$ of expression-matched proteincoding genes expressed in each tissue were eGenes suggests that there are other factors involved. One such factor could be simpler regulatory mechanisms, which have been reported in certain types of lncRNAs (19). With fewer modifiers of their expression, eQTLs for IncRNA genes may 
occur less frequently, but have stronger effects when present. This is supported by our observation that lead eVariants (the genetic variants with the most significant associations for each gene) for lncRNA genes had larger effect sizes than expression-matched protein-coding genes, with a median $\log _{2}$ (allelic fold-change) of 0.805 for lncRNA lead eVariants and 0.579 for expression-matched protein-coding lead eVariants (Wilcoxon rank-sum test $\mathrm{W}=4.88 \times 10^{9}, \mathrm{p}$ $<2.2 \times 10^{-16}$; Fig. 2B) (20). Higher effect sizes were also reported in a previous study of intergenic long non-coding RNA (lincRNA) eQTLs compared to protein-coding genes, which the authors attributed to less constraint on lincRNA gene expression (21). Intriguingly, however, the proportion of eGenes with $>1$ independent eQTL is comparable between lncRNA and proteincoding eGenes (Fig. 2C).

The tissue-specificity of eGenes across all gene groups (Fig. 2D) follows the patterns of tissue-specificity in gene expression (Fig. 1D), with lncRNA eGenes generally observed in fewer tissues than protein-coding eGenes. There were more broad tissue-specific eGenes than there were genes with broad tissue-specific expression: 2,783 lncRNA eGenes, and 1,267 proteincoding eGenes (Fig. 2E; Table S3). Given the similar patterns of tissue-specificity in both gene expression and the presence of eGenes, a natural assumption would be that many of the tissuespecific eGenes are simply genes with tissue-specific expression that have eQTLs. Surprisingly, this was rarely the case: except for the testis-specific eGenes and prostate-specific lncRNA eGenes, fewer than $25 \%$ of tissue-specific eGenes also showed tissue-specific gene expression (median overlap 4.5\%) (Fig. S3).

Previous studies have found that different subtypes of lncRNA genes have different promoter structure and expression patterns $(1,19)$. Of the GENCODE IncRNA biotypes, we noted that $62 \%$ of lncRNAs with tissue-specific expression were lincRNAs, which was higher than their proportion in total lncRNAs $\left(53 \% ; \chi^{2}=142.5, \mathrm{p}<2.2 \times 10^{-16}\right.$, test of equal proportions) (Fig. S4A). In contrast, antisense lncRNAs were depleted for tissue-specific expression: $31 \%$ of IncRNA genes with tissue-specific expression were antisense, compared to $37 \%$ of the total set $\left(\chi^{2}=66.2, p=4.1 \times 10^{-16}\right.$, test of equal proportions). This is consistent with previous reports of lincRNAs having notably high tissue-specificity in comparison to lncRNA genes that diverge from another gene (via bidirectional transcription) (19), since many of the GENCODE-annotated antisense lncRNA genes are identified to actually be promoter-divergent in the FANTOM CAGE-associated transcriptome (FANTOM-CAT) (1) (Fig. S4B). It was also interesting to note that $15 \%$ of GENCODE intergenic lncRNAs were identified as promoter-divergent in FANTOM-CAT. This highlights the importance of maintaining updated gene annotations, especially when examining the frequently updated lncRNA genetic landscape (22).

\section{Multi-tissue outliers for intergenic IncRNA gene expression are frequently overexpressed}

Given that lncRNA regulation is often tissue-specific, we were interested in whether individuals can defy these patterns and display outlier lncRNA gene expression in non-canonical tissues. To test this, we confined the outlier gene expression analysis performed in the GTEx rare variants paper (23) to only examine lincRNA genes, the lncRNA gene subtype that most commonly displayed tissue-specific expression (Fig. S4A). To identify multi-tissue outliers, we also limited our analysis to individuals with expression data available for the given gene in at least 5 tissues. Overall, 2,535 individual-lincRNA combinations were found to be expressed at more than 2 standard deviations above or below the mean in a majority of tested tissues; these 
were termed multi-tissue outliers ( $\mid$ median Z-score| > 2) (Table S4). These outlier events (with an event being an individual-lincRNA combination) involved 1,009 unique lincRNAs out of the 4,236 tested. The majority of lincRNA outliers (86\%) have a positive median Z-score, which means that the outlier individuals typically over-expressed that lincRNA gene (Fig. 3A). For protein-coding genes, only $61 \%$ of outliers at the same threshold are over-expressed (23). This is mostly attributable to the lower expression of lincRNA genes compared to protein-coding genes; lowly expressed genes are more likely to fluctuate upward, and their under-expression is difficult to detect.

For each outlier, we identified variants within $10 \mathrm{~kb}$ of the outlier gene for individuals with self-reported European ancestry, as allele frequencies may not be consistent across populations (23). Outlier individuals were more enriched for nearby variants with lower allele frequencies (MAF < 1\%; see Methods), with relative risks (RRs) of 1.12 for SNPs, 1.28 for indels, and 9.56 for SVs (Fig. 3B). In both over- and under-expression outliers, higher proportions of nearby rare variants were observed compared to non-outliers $(54.4 \%$ of overexpression outliers, $50 \%$ of under-expression outliers, and $47 \%$ of non-outliers), though the proportion of overall rare variants nearby under-expression outliers vs non-outliers was not significantly enriched $\left(\mathrm{RR}\right.$ over $=1.16, \mathrm{p}=2.82 \times 10^{-9}, \mathrm{RR}$ under $=1.06, \mathrm{p}=0.37$, Wald test $)$. Rare structural variants were more often associated with under-expression of the lincRNA (RR = $10.53, \mathrm{p}=5.59 \times 10^{-20}$, Wald test), and rare variants in transcription start sites (TSS) were enriched in both directions (Fig. 3C). Of the rare structural variants driving the enrichment nearby outliers (Fig. 3B), we found that deletions, CNVs, and duplications were specifically enriched in outlier individuals near their outlier genes (Fig. 3D). However, rare splice variants were also strongly enriched nearby outlier genes $\left(R R=6.78, p=5.20 \times 10^{-8}\right)$ - even more so than rare TSS variants $\left(\mathrm{RR}=2.92, \mathrm{p}=4.35 \times 10^{-26}\right)$. This indicates that transcript structure is a key influence on overall expression; splicing variation may mediate this by affecting transcript maintenance and decay. This is also supported by the similarly strong enrichment for rare splice variants observed near protein-coding gene outliers (23), as well as the strong enrichment of splice-related annotations for $c i s$-eQTLs, including those that were distinct from splice QTLs (18).

We next investigated how many multi-tissue outliers disrupted the tissue-specific patterns of lincRNA gene expression. Of the 2,535 outliers, 675 involve lincRNAs that were only expressed in 1-5 of the 49 GTEx tissues (301 unique genes). Per-tissue Z-scores were lower in the tissues that typically expressed these outlier genes compared to those that did not (median in expressing tissues $=2.1$, median in non-expressing tissues $=2.5$, Wilcoxon rank-sum test $\mathrm{p}$ $<2.2 \mathrm{e}-16$ ) (Fig. 3E). This suggests that outlier events involving tissue-specific lincRNAs could have particularly dramatic effects by involving aberrant expression in tissues that do not usually express the lincRNA. This occurred in 296 outlier events in which lincRNAs with tissue-specific expression had TPM $\geq 0.5$ in at least one non-canonical tissue (in which the lincRNA gene's TPM $<0.1$ in $>80 \%$ of samples) (Fig. 3F).

One notable outlier involves the gene RP11-276M12.1 (ENSG00000259445). This lincRNA gene was typically expressed in three tissues: thyroid, testis, and vagina. There were 13 over-expression outlier individuals for this gene, of whom 12 were assessed for the presence of rare variants. Of these 12 individuals, 11 had rare variants nearby the gene, and 8 of these individuals had the same rare variant located in the first exon, within 100 bases of the TSS (Fig. $3 G)$. RP11-276M12.1 was expressed at TPM $\geq 0.5$ in 0 to 4 non-canonical tissues, depending on 
the outlier individual (median =1). This was spread across eight different tissues, with tibial artery showing non-canonical expression of the gene in eight outlier individuals. The individual who expressed $R P 11-276 M 12.1$ at TPM $\geq 0.5$ in four different non-canonical tissues did so in the two cerebellum tissues, spinal cord (cervical-C1), and cultured fibroblast cells. This gene is interesting not just because of the high number of outlier individuals, but also that many of them share the same rare variant. This variant, a $\mathrm{G}>\mathrm{C}$ substitution at chromosome 15 position 81,995,598 (hg38 assembly), has a frequency of 0.0063 in GTEx and 0.0066 in gnomAD nonFinnish Europeans ( 0.0045 overall). It is associated with a significant difference in expression (Wilcoxon rank-sum test $\mathrm{p}=1.2 \mathrm{e}-06$; Fig. 3G), but was missed by traditional eQTL testing that filters out variants with MAF $<0.01$.

\section{eVariants can be shared between IncRNA and protein-coding genes}

Since many lncRNA genes have cis-regulatory effects on other nearby genes (24), we assessed the prevalence of shared genetic effects. Across all independent lead eVariants, $7.5 \%$ were associated with $>1$ gene in the same tissue (Fig. 4A). The other $92.5 \%$ were only associated with 1 gene, most of which were protein-coding genes $(65.2 \%$, compared to $17.3 \%$ for lncRNAs and $10.0 \%$ for all other gene types).

When the shared eVariants were broken down by which types of genes shared them, a notable proportion $(26.3 \%$ ) were shared between protein-coding and lncRNA genes, which was second only to the proportion shared between multiple protein-coding genes $(32.8 \%)$. This high occurrence of shared eVariants with lncRNA genes may be partly driven by both antisense-sense gene pairs (13.4\% of protein-coding-lncRNA gene pairs that share an eVariant are antisensesense gene pairs), cis-regulatory relationships or shorter distances between lncRNA and proteincoding gene pairs (Fig. 4B).

Of the 26,469 gene pairs sharing eVariants in at least one tissue, 3,568 (13.5\%) had cross-mappability scores greater than zero (24). Higher cross-mappability scores between gene pairs indicate a greater amount of sequence similarity, and thus greater potential for incorrect read alignment to have affected quantification. Our observed percentage of cross-mapping pairs is higher than the 2.45-4.92\% of evaluated gene pairs in the GTEx v7 dataset that were reported as cross-mappable (25), suggesting that some of the eVariant sharing may actually be due to cross-mapping. Although the eVariant-sharing gene pair types involving lncRNA genes actually have the lowest proportions of cross-mappable gene pairs (Fig. S5A), this is still an important technical factor to consider; as such, we reported the symmetric cross-mappability score results for all eVariant-sharing gene pairs (Table S5).

Several lncRNA genes shared eVariants across multiple tissues. One such gene was KANSL1-AS1 (ENSG00000214401), which was connected to 5 different protein-coding genes across many tissues, with the genes sometimes even sharing more than one eVariant (Fig. S5B). These recurring connections may highlight key sets of co-regulated genes.

\section{Most cis-regulation by IncRNA genes operates on a local scale}

One of the best-known lncRNA genes, XIST, operates in cis on a massive scale, inhibiting almost the entire X-chromosome from which its expressed $(26,27)$. We were curious about other lncRNA genes' range of local regulation, which we assessed via allele-specific 
expression (ASE). We defined genes with strong ASE (multiple test-adjusted binomial p-value $\leq 0.05$ and allele ratio either $0.02-0.15$ or $0.85-0.98$ for any variant in the gene) as "central genes", and then tracked how often significant ASE (multiple test-adjusted binomial p-value $\leq 0.05$, no allele ratio threshold) was maintained in the same tissue in non-overlapping, proteincoding gene neighbors.

Extending out from both protein-coding and lncRNA central genes, over half of all nonoverlapping protein-coding gene neighbors also showed significant ASE, with the degree of sharing decreasing as the distance between the genes increased. Nearby downstream neighbors of lncRNA genes had the highest proportions of ASE, and the drop-off in ASE maintenance with distance was greatest from lncRNA central genes (Fig S6A), potentially reflecting antisensesense gene pair relationships or other mechanisms for cis-effects, such as localized epigenetic changes.

Pairs of genes that shared eVariants were more likely to share ASE, compared to central genes and gene neighbors with unshared eQTLs $\left(\chi^{2}=157.7\right.$ for lncRNA central genes and 403.4 for protein-coding central genes, both $\mathrm{p}<2.2 \times 10^{-16}$, test of equal proportions) (Fig. $4 \mathrm{C}$ ). For lncRNA central genes, this relationship appeared to be dependent on distance. Altogether, these patterns of ASE suggest that most lncRNA genes operate differently than XIST, and tend to affect the genes immediately around themselves, if at all, rather than have more far-reaching effects. One interesting example of local ASE was with the lncRNA gene RP4-568C11.4 (ENSG00000274173), in which 52/59 individuals also displayed ASE in two nearby proteincoding genes (Fig. S6B). Remarkably, for all of these individuals the significant ASE occurred in the same tissue (whole blood).

Despite these overall patterns, there were some examples of lncRNA genes surrounded by large regions of ASE. One such example was the central lncRNA gene MIR210HG (ENSG00000247095), for which ASE was seen in protein-coding genes over a range of $358 \mathrm{~kb}$ downstream to $444 \mathrm{~kb}$ upstream of the gene in 48 individuals (Fig. S6C).

\section{Co-expression networks identify highly connected lncRNA genes with specialized cell type associations}

For each tissue, we built co-expression networks using weighted correlation gene network analysis (WGCNA) (28). Since the goal was to identify lncRNA genes with cell type associations, each module was annotated based on enrichment for gene sets associated with Gene Ontology Cellular Compartments and cell types from blood, the central nervous system, and the Mouse Cell Atlas (29) (see Methods). The number of modules created per tissue was not associated with the sample size of the tissue (Fig. S7A). A median of $48 \%$ of modules in each tissue were annotated, ranging from $18 \%$ of modules in the ovary to $85 \%$ of modules in the stomach (Fig. S7B).

With the exception of testis tissue, approximately $50 \%$ of lncRNAs did not meet the expression requirements to be included in the co-expression networks (median of excluded lncRNA genes across tissues $=47 \%)($ Fig. 5A). The proportion of excluded genes was lower for lncRNA genes with known function (median $=35 \%$ ). Greater proportions of protein-coding genes (both the total group and the expression-matched group) were assigned to modules compared to lncRNA genes (Fig. 5A). Generally, larger modules included higher proportions of 
lncRNA genes (Fig. 5B); however, there were also some smaller modules mostly made up of lncRNA genes, which may be worthy of further exploration as potential hubs of lncRNA regulatory activity in those tissues.

We evaluated how strongly connected genes were based on their intramodular connectivity $\left(\mathrm{k}_{\text {in }}\right)$ values, which were converted to a rank and grouped into deciles within each module. Higher proportions of protein-coding genes were among the highest-ranked (most connected) genes, although this was expression-dependent (Fig. 5C). The majority of lncRNA genes in each module were not highly connected, indicating that most lncRNAs only have potential regulatory relationships with one or few nearby genes. However, although they were in the minority, there were also some highly connected lncRNA genes that may represent lncRNAs with potentially far-reaching regulatory effects (Table S6). We also incorporated an "all other gene types" group, which included all non-coding genes that are not lncRNAs as well as pseudogenes. Since many of the quantifications in this group are of poor quality, it served as a measure of noise in the networks. As expected, lncRNAs were generally better connected than this "all other gene types" category (Fig. $5 \mathrm{C} ; \mathrm{p}<2.2 \times 10^{-16}$, Wilcoxon rank-sum test of decile rankings between the "total lncRNA genes" group and "all other genes" group). With module membership values, which reflect how well a gene's expression correlates with its assigned module, the same trend was observed of protein-coding genes having the highest scores and lncRNA genes having lower module membership, but not as low as the catch-all "all other gene types" category (Fig. S7C).

We next examined the most common annotations of highly connected genes. To be considered "highly connected", a gene had to be within the top decile of its module based on $k_{\text {in }}$ values and have a scaled $\mathrm{k}_{\text {in }}$ value $\geq 0.5$. Of the highly connected lncRNAs $(5,141$ gene-tissue combinations), only 1,960 were assigned to annotated modules (Fig. 5D). The high proportion of well-connected lncRNA genes in unannotated modules suggests that many of the pathways involving lncRNAs have yet to be identified.

Compared to the modules that protein-coding genes were assigned to, a higher proportion of lncRNA annotation terms related to specialized cell types such as secretory alveoli cells of lungs, B cells of the immune system, pit cells of the liver, spermatocytes, and kidney regions like tubule cell, loop of Henle, and distal convoluted tubule (Fig. 5D, Fig. S7D). In contrast, proteincoding genes were more frequently assigned to modules annotated with common cell types and ubiquitous cell compartments terms such as endothelium, resident macrophages, mitochondria, and nucleolus. Although lncRNA genes rarely formed the hubs of a given module, the annotation of these networks provides a resource to identify cell type or compartment-related lncRNA 35 genes.

There were $>1.5 \times 10^{6}$ unique lncRNA/protein-coding gene pairs that shared modules in $>12$ tissues, which was $3 *$ IQR of module sharing across all lncRNA-coding gene pairs (Fig. 5E; Table S7). 614 of these gene pairs also shared at least 1 multi-tissue outlier individual for both genes (Fig. S7E). Of these 614 co-expressed genes with shared outliers, 71 of them involved a rare variant within $10 \mathrm{~kb}$ of the lncRNA gene (Fig. S7F). These 614 gene pairs are compelling candidates to explore further for functional links.

There were some notable candidate lncRNA/protein-coding gene pairs due to a high degree of module sharing $(\mathrm{N}>12)$ and multiple outlier events. The first pair, MICA and AL645933.2 (ENSG00000272221), were both over-expression outliers in two individuals, and 
both under-expression outliers in one individual. One of these individuals had different rare SNVs within $10 \mathrm{~kb}$ of both genes (Fig. 5F). MICA encodes major histocompatability complex class I chain-related protein A, a highly polymorphic stress-induced antigen that is associated with inflammatory responses and cancer (30-32). The co-expression of AL645933.2 with MICA suggests that it may also have a role in these processes, although 13 of the modules they share across 19 tissues are unannotated. Another gene pair, ACOT1 and AC007228.9 (ENSG00000268568), was intriguing because they are present on separate chromosomes. These genes shared two under-expression outlier individuals. In both cases, the individual had a rare SNV within $10 \mathrm{~kb}$ of $A C 007228.9$. ACOT1, which encodes acyl-CoA thioesterase 1, is involved in lipid metabolism. Given that these two genes' shared modules were frequently annotated as mitochondria modules, as well as being assigned to the adipocyte module in the adipose (visceral omentum) tissue, AC007228.9 may also be involved in lipid metabolism.

\section{The majority of significant IncRNA colocalization events have a stronger signal than nearby protein-coding genes}

Colocalization analyses connect genetic variation, gene expression, and traits by integrating results from eQTL analyses and genome-wide association studies (GWAS). As part of the main GTEx paper (18) and GWAS companion paper (33), colocalization analyses were performed for 87 traits in each tissue. There were 4,694 significant events (posterior probability of a shared predicted causal variant between eQTL and GWAS (PP4) $\geq 0.5$ ) involving lncRNA genes, which encompassed 48 traits and 690 lncRNA genes (Table S8). As a point of reference, there were 20,281 significant protein-coding gene colocalization events, involving 53 traits and 2,785 unique genes.

For most of the lncRNA gene colocalization events $(3,757,80.0 \%)$, there was no significant protein-coding gene colocalization within $500 \mathrm{~kb}$ up or downstream of the lncRNA gene (Fig. 6A). This is not due to an absence of in-range protein-coding genes; there were only 32 events $(0.7 \%)$ in which there was no annotated protein-coding gene within this range, and those events were excluded from Fig. 6A. Of the lncRNA colocalizations that also had a significant in-range protein-coding gene colocalization, most pairs had similar PP4 values (479, $10.2 \%$ ), followed by a nearly even split between events with a notably higher protein-coding gene PP4 $(239,5.1 \%)$ and ones with a notably higher lncRNA gene PP4 (187, 4.0\%). Compared to the total set of lncRNA genes, lincRNA genes alone had an even greater proportion of colocalization events with no in-range significant protein-coding gene, reflecting their independent regulation. In contrast, fewer colocalization events involving antisense lncRNAs had no in-range significant protein-coding genes.

Across all significant colocalization events, lncRNA genes were depleted compared to their proportion in the gene set tested (Fig. 6B). However, the proportion of lncRNA colocalization events varied by trait type, with the highest percentages occurring in migraine (31\%), and psoriasis (50\%). For psoriasis, colocalization events were dominated by one lncRNA gene. The single-exon lncRNA C6orf3 (ENSG00000255389), which runs sense intronic to the protein-coding gene TRAF3IP2, showed significant colocalization in 35 tissues. No proteincoding genes in the $1 \mathrm{Mb}$ neighborhood around C6orf3 had a significant colocalization with psoriasis in any tissue (Fig. S8A). The tissue with the highest PP4 value (0.996) was sun-exposed skin, which is compelling given that psoriasis is a chronic skin condition. Notably, top 
significant eQTLs for C6orf3 are the exact top GWAS variants, which does not always occur with colocalization (Fig. S8B). In both skin tissues (sun-exposed and not sun-exposed), C6orf3 clustered in the "endothelial cell"-annotated co-expression module (Fig. S7C).

For significant lncRNA colocalizations, the in-range protein-coding gene with the highest PP4 was not always the closest gene (Fig. S8A). This indicates that many of these connections (i.e. the sharing of a significant colocalization) were not just the result of proximity, but may also reflect some regulatory relationship. For the events in which there was no in-range proteincoding gene with a significant colocalization, this connection was essentially arbitrary, since the protein-coding genes' PP4 was $<0.5$ in these cases. Thus, these lncRNA/protein-coding gene pairings have limited relevance, which is reflected in the wide range of distances between the two genes (Fig. S8A). It is also worth noting that, for 1,435 of the 3,757 events with no in-range significant protein-coding gene, the significant lncRNA gene actually overlapped a proteincoding gene, but it still did not have a significant colocalization. For example, this was the case with C6orf3 and TRAF3IP2.

Another interesting case involved the lncRNA genes LINC01475 (ENSG00000257582) and RP11-129J12.1 (ENSG00000228778) and the protein-coding gene $N K X 2-3$ in relation to ulcerative colitis. The two lncRNA genes have antisense overlap with each other, and are just upstream from $N K X 2-3$ (Fig. 6Ci). NKX2-3 has received attention related to the significant colitis GWAS results in this genomic region (34). However, it did not have a significant colocalization in any tissue, whereas the two lncRNA genes had the best colocalizations in the transverse colon (PP4 $=0.749$ for both genes) and the spleen (PP4 = 0.732 for both genes) (Fig. 6Cii, Fig. S9B).

Both of the tissues in which colocalization occurred are logical for ulcerative colitis: the colon has an obvious role, and the spleen could be connected via immune system regulation. In the co-expression network for the transverse colon, all three genes were assigned to the same "smooth muscle cell" module, which was unsurprising given their correlated gene expression (Fig. S9C). In the spleen co-expression network, NKX2-3 was assigned to one "endothelial cell" module, and the two lncRNA genes were assigned to a different "endothelial cell" module (Fig. 6Ciii-iv). NKX2-3 is a homeobox gene that is key for the development of the spleen and the visceral mesoderm, which develops several essential cell types of the gastrointestinal tract including endothelial cells, immune cells, and - notably - smooth muscle cells. Knockout mouse studies have shown that loss of this gene affects spleen architecture, and lymphocyte maturation and homing (35-39). These findings make a compelling case that lncRNA regulation of NKX2-3 in both the colon and spleen influences ulcerative colitis susceptibility.

\section{Discussion}

lncRNA genes differed from protein-coding genes at nearly all levels of regulation. Compared to protein-coding genes, lncRNAs showed greater tissue-specificity in both expression and presence of eQTLs, with the latter not entirely attributable to differences in expression levels, as well as lower intramodular connectivity in gene co-expression networks. Setting lncRNA genes apart even more was the striking number of significant lncRNA colocalization events where there was no significant protein-coding colocalization within $1 \mathrm{Mb}$ $(3,757 / 4,694,80 \%)$. There were often differences between lncRNA subtypes as well: intergenic lncRNA genes more frequently showed tissue-specific expression and significant colocalization events with no nearby significant protein-coding gene colocalizations, whereas antisense 
lncRNA genes more frequently shared eVariants with other genes. These subtype-specific trends show the importance of maintaining updated lncRNA gene annotation, and interpreting them cautiously. For instance, many lncRNA subtype assignments did not coincide between FANTOM-CAT and GENCODE (1). In the current version of GENCODE (v31), they have dispensed with subtype categorization entirely, referring to them all as gene type 'IncRNA'. It is clear that different subtypes of lncRNA genes have different regulatory patterns and perhaps different roles, and subtype should be considered in any analysis of these genes.

Not only do these analyses highlight the differences between lncRNA and protein-coding genes, but they can also be used to interrogate lncRNA genes of interest and systematically identify lncRNAs associated with certain cell types or traits. There are other resources that provide lncRNA gene networks, conservation data, or expression-based assessments $(11,40-43)$; our analyses examine several of these characteristics, and also provide trait association information. Searching for convergence of evidence across multiple lncRNA resources here enabled identification of the most compelling candidate genes for further study.

Although exploring non-coding genetic variation has become increasingly important, efforts to date have mostly focused on regulatory effects on protein-coding genes. This work provides an important pathway to enhance these efforts towards evaluating non-coding genes and their roles in complex traits and diseases. 
bioRxiv preprint doi: https://doi.org/10.1101/793091; this version posted October 4, 2019. The copyright holder for this preprint (which was not certified by peer review) is the author/funder, who has granted bioRxiv a license to display the preprint in perpetuity. It is made available under aCC-BY-NC 4.0 International license.

A

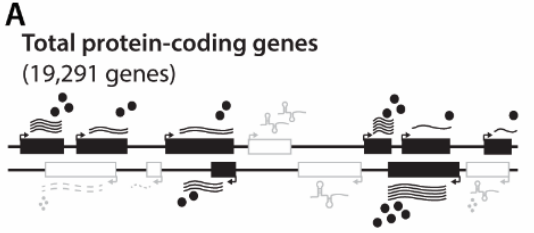

Expression-matched protein-coding genes $(15,410$ genes $)$

Total IncRNA genes

(14,100 genes)

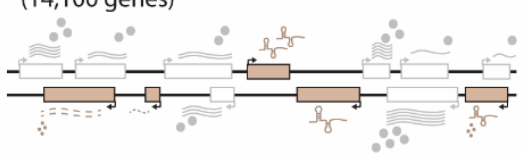

High-confidence non-coding IncRNA genes (4,510 genes)

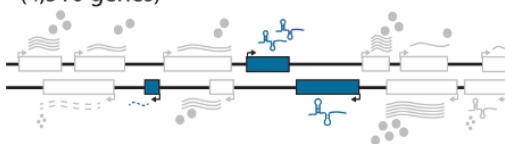

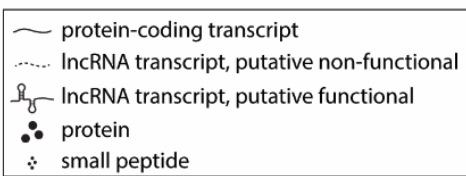

IncRNA genes with known function (713 genes)

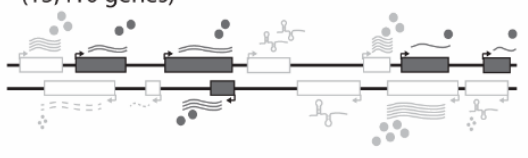

B

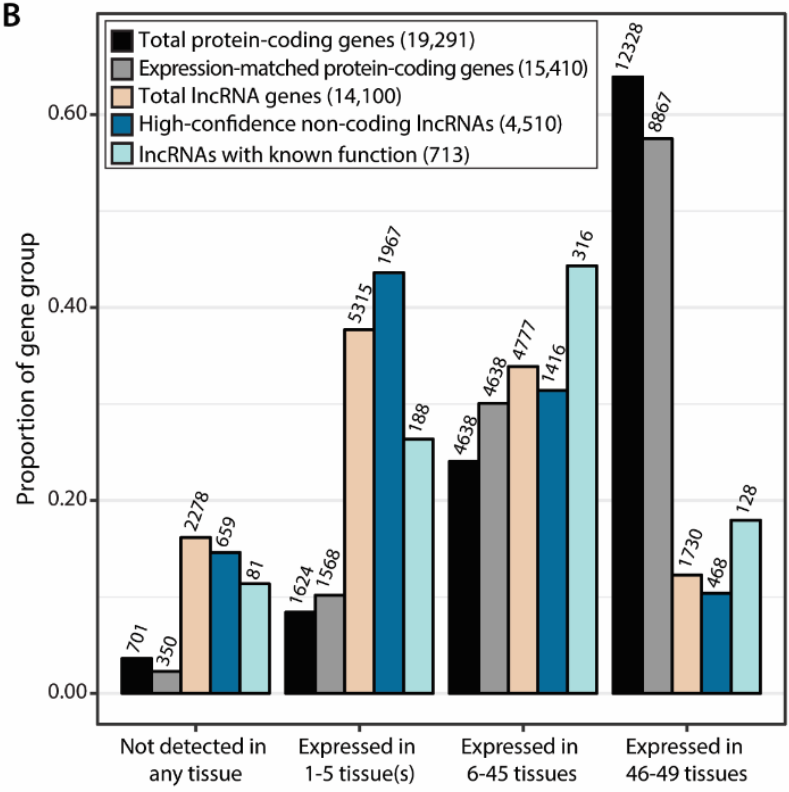

$\mathrm{C}$
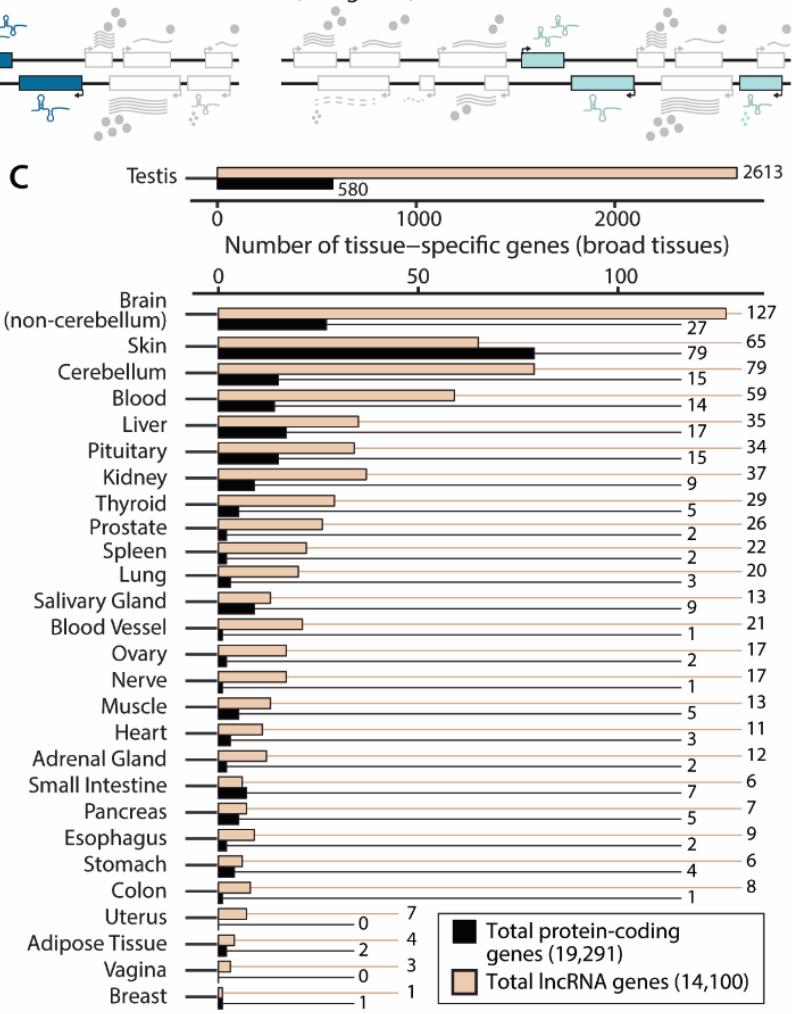

Fig. 1. Tissue-specificity of lncRNA and protein-coding gene expression in GTEx. (A) The protein-coding and IncRNA gene groups compared in this paper. The "Expression-matched protein-coding genes" group is a subset of the "Total protein-coding genes", and the "High-confidence non-coding lncRNA genes" and "IncRNA genes with known function" groups are subsets of the "Total lncRNA genes". (B) Proportion of each gene group expressed in a certain number of tissues. Bar labels show the number of genes. (C) Numbers of lncRNA and protein-coding genes expressed in only one of the 28 broad tissues. For (B) and (C), the expression threshold is TPM $\geq 0.5$ in $>20 \%$ of samples. 
A

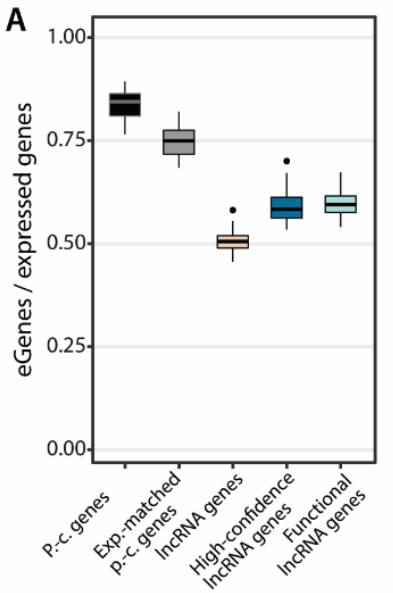

D

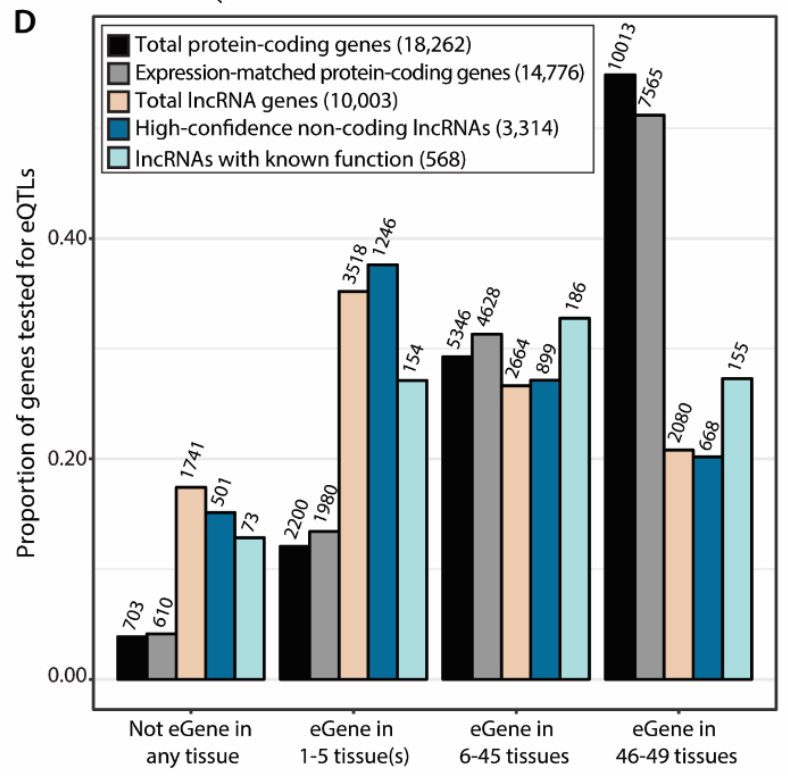

B

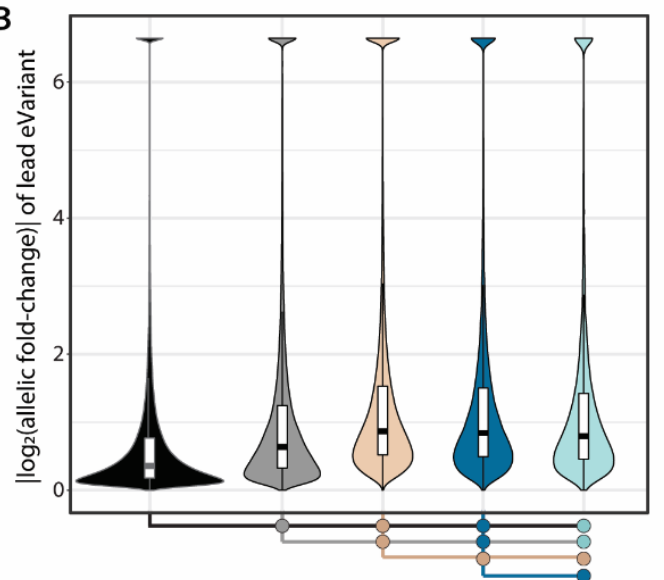

E

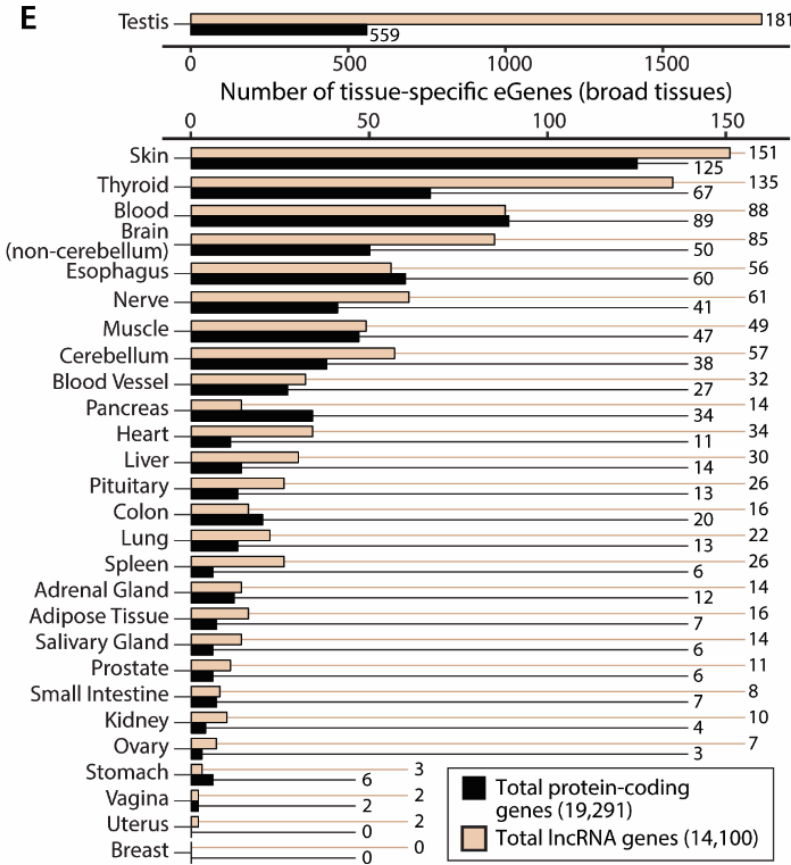

C Protein-coding eGenes

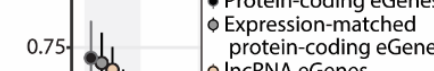
IncRNA eGenes 巴) 巴) 0.50 年 0.25 . แक्र
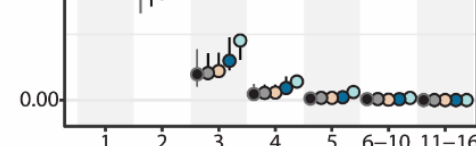

5 6-10 11-16 814 


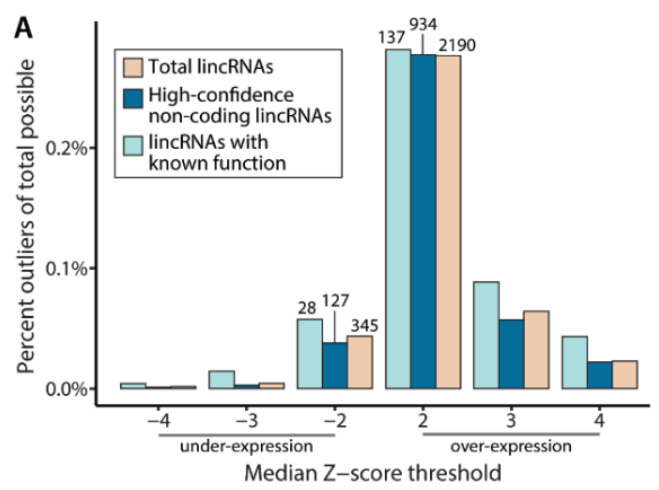

C

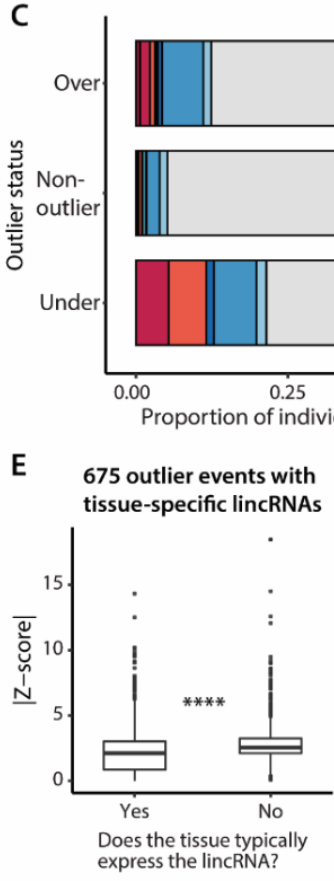

B

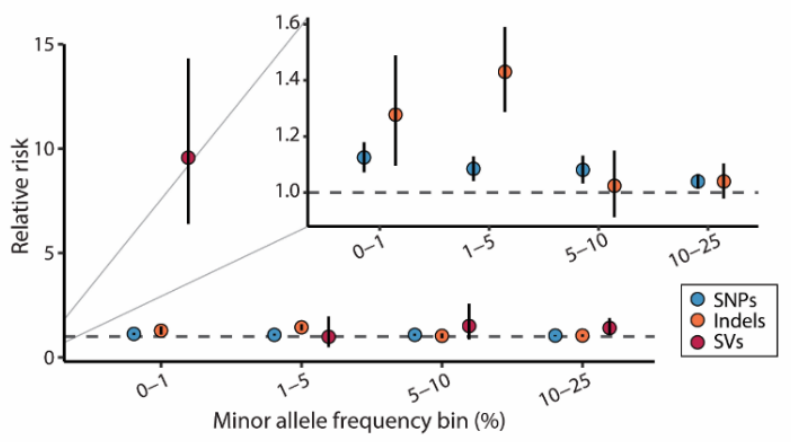

D

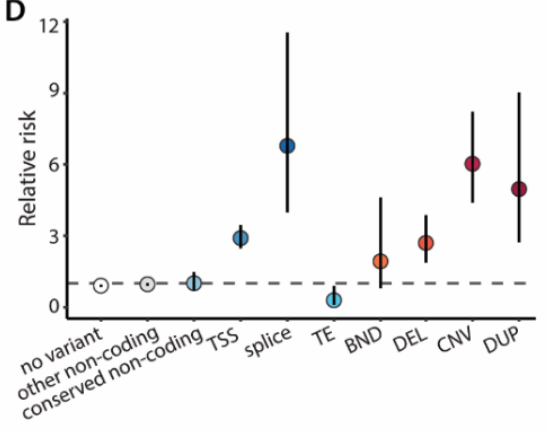

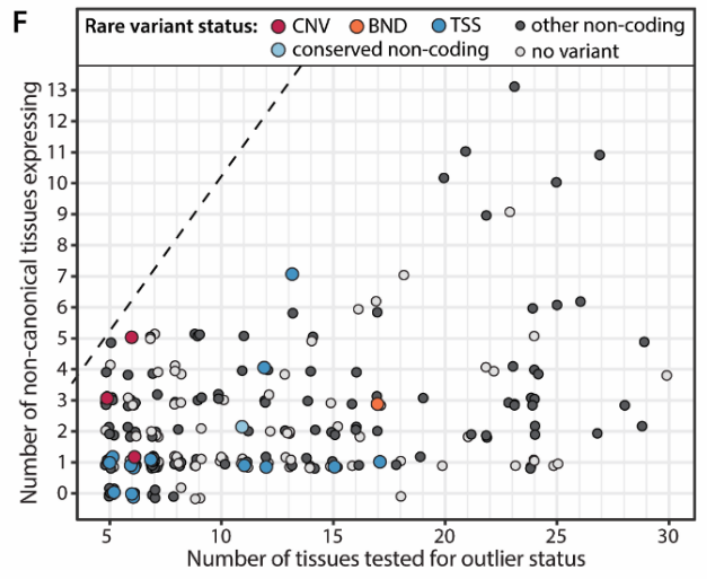

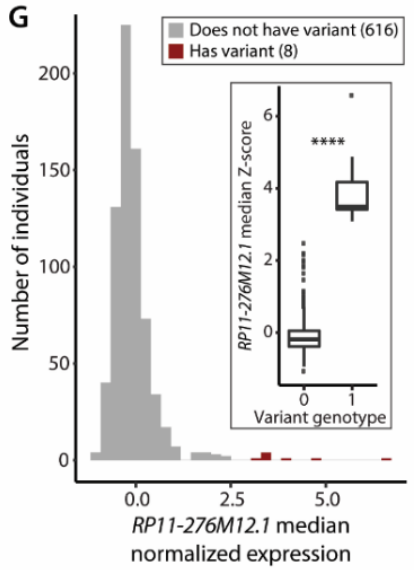

Fig. 3. Outliers in lincRNA expression. (A) Percent of multi-tissue lincRNA gene outliers (gene-individual combinations) out of all gene-individual combinations tested. Labels indicate the number of outliers. (B) Enrichment of variants within $10 \mathrm{~kb}$ of the outlier gene in outlier individuals. (C) Presence of rare variants (MAF $<1 \%$ ) within $10 \mathrm{~kb}$ of the outlier gene based on outlier status. (D) Enrichment of rare variants (MAF <1\%) within $10 \mathrm{~kb}$ of the outlier gene in outlier individuals. (E) Tissue-specific Z-scores for outlier events involving lincRNAs with tissuespecific gene expression (as identified in Fig. 1), separated by whether or not the tissue typically expresses that lincRNA. (F) For outlier events involving tissue-specific lincRNA genes, the number of non-canonical tissues expressing the gene in the outlier individual versus the number of their tissues that were tested for outlier status. Non-canonical expression was TPM $\geq 0.5$ in the outlier individual's sample, for a tissue had TPM $<0.1$ in $>80 \%$ of samples. Calling non-canonical expression was not limited to the tissues that were tested for outliers (so an individual could have more tissues with non-canonical expression than tissues tested). (G) Normalized expression of RP11-276M12.1, a gene that was a multi-tissue outlier for 13 individuals. Each value on the histogram is one individual's median expression of the gene across all tissues. Of these individuals, 8 had the same rare variant in the first exon (filled in red). Inset: Individuals' median Z-scores for this gene separated by presence or absence of the rare variant. $\mathrm{DUP}=$ duplication, $\mathrm{CNV}=$ copy number variation, $\mathrm{DEL}=$ deletion, $\mathrm{BND}=$ breakend, $\mathrm{TE}=$ transposable element insertion, TSS $=$ transcription splice site. 

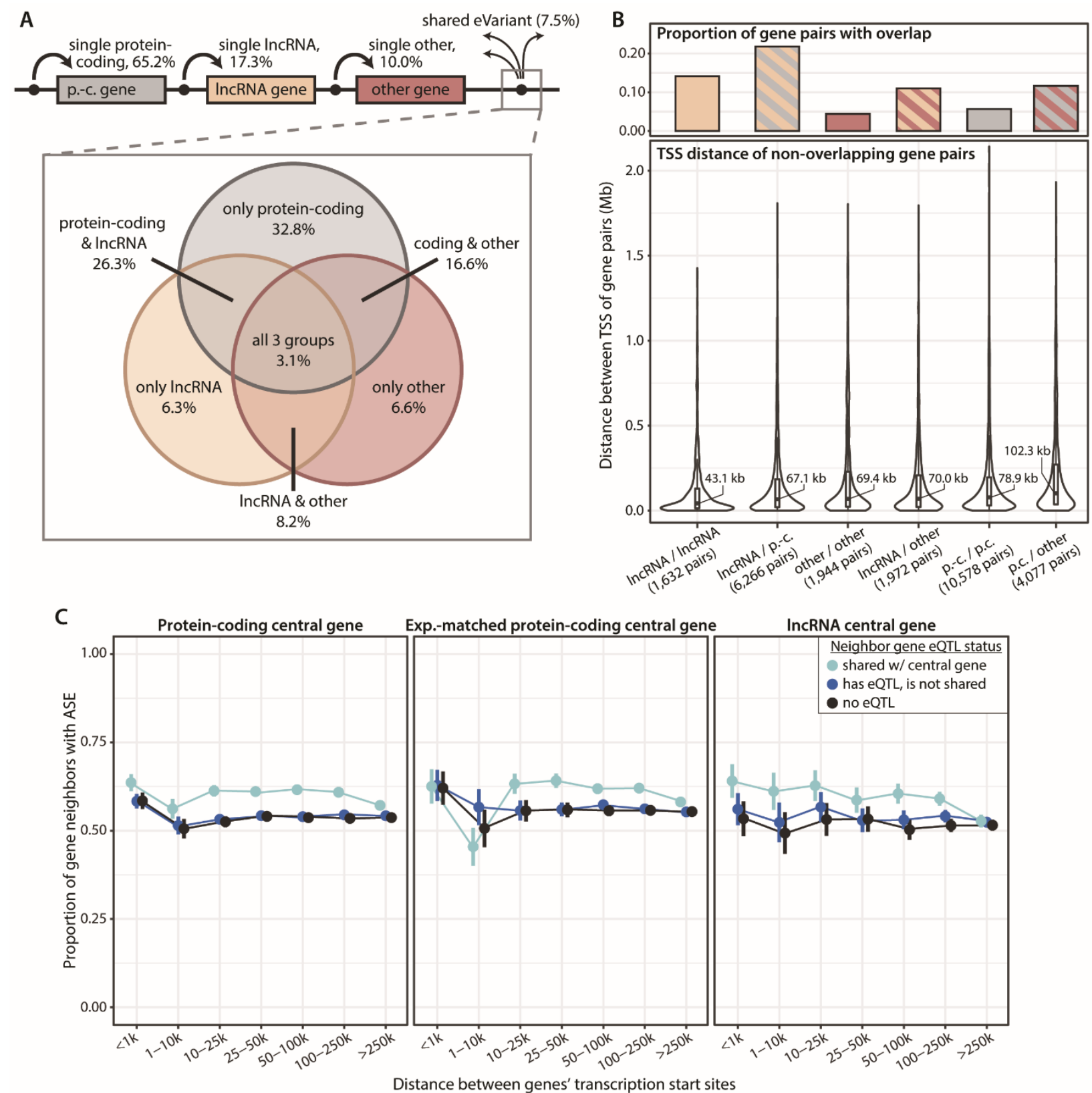

Fig. 4. Connecting genes through shared eVariants. (A) Summary of eVariant sharing using the independent eQTLs (see Methods). Genes shared an eVariant if their expression was associated with either the same eVariant, or with eVariants that were within $500 \mathrm{~kb}$ of each other with an $\mathrm{R} 2 \geq 0.85$, in the same tissue. (top) percentage of eQTLs only associated with one gene ("single") versus how many were associated with $>1$ gene ("shared"). (bottom) The shared eVariants categorized based on which types of genes they were associated with. (B) (top) Proportion of eVariantsharing gene pairs that have overlapping genomic location. (bottom) Distance between TSS's of non-overlapping gene pairs that share an eVariant. (C) Extension of allele-specific expression (ASE) from a central gene with strong ASE (adjusted binomial p-value $\leq 0.05$, allelic ratio deviation from 0.5 of $0.35-0.48$ ). The line colors indicate whether the neighboring gene has an eQTL, and whether its associated variant is shared with the central gene. Neighboring genes were limited to non-overlapping protein-coding genes only, and were checked for significant ASE (adjusted binomial p-value $\leq 0.05$ ). Bars indicate the $95 \%$ CI across different central genes, with ASE status being collapsed by individuals and tissues. p.-c. gene $=$ protein-coding gene . 
bioRxiv preprint doi: https://doi.org/10.1101/793091; this version posted October 4,2019 . The copyright holder for this preprint (which was not certified by peer review) is the author/funder, who has granted bioRxiv a license to display the preprint in perpetuity. It is made available under aCC-BY-NC 4.0 International license.
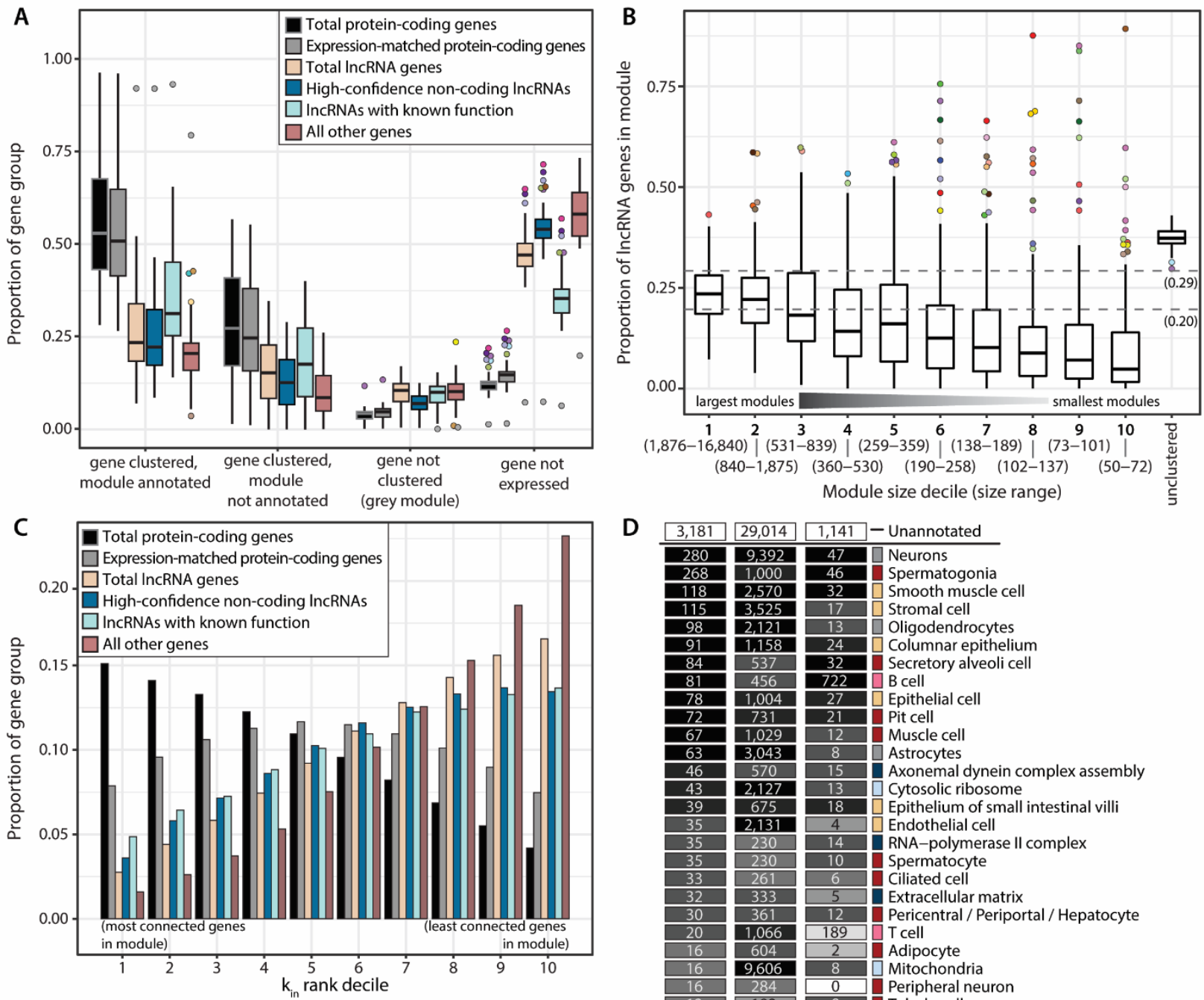

\section{D}

\begin{tabular}{|c|c|c|c|}
\hline 3,181 & 29,014 & 1,141 & - Unannotated \\
\hline 280 & 9,392 & 47 & Neurons \\
\hline 268 & 1,000 & 46 & Spermatogonia \\
\hline 118 & 2,570 & 32 & Smooth muscle cell \\
\hline 115 & 3,525 & 17 & Stromal cell \\
\hline 98 & 2,121 & 13 & Oligodendrocytes \\
\hline 91 & 1,158 & 24 & Columnar epithelium \\
\hline 84 & 537 & 32 & Secretory alveoli cell \\
\hline 81 & 456 & $\overline{722}$ & B cell \\
\hline 78 & 1,004 & 27 & Epithelial cell \\
\hline 72 & 731 & 21 & Pit cell \\
\hline 67 & 1,029 & 12 & Muscle cell \\
\hline 63 & 3,043 & 8 & Astrocytes \\
\hline 46 & 570 & 15 & Axonemal dynein complex assembly \\
\hline 43 & 2,127 & 13 & Cytosolic ribosome \\
\hline 39 & 675 & 18 & Epithelium of small intestinal villi \\
\hline 35 & 2,131 & 4 & Endothelial cell \\
\hline 35 & 230 & 14 & RNA-polymerase II complex \\
\hline 35 & 230 & 10 & Spermatocyte \\
\hline 33 & 261 & 6 & Ciliated cell \\
\hline 32 & 333 & 5 & Extracellular matrix \\
\hline 30 & 361 & 12 & Pericentral / Periportal / Hepatocyte \\
\hline 20 & 1,066 & 189 & T cell \\
\hline 16 & 604 & 2 & Adipocyte \\
\hline 16 & 9,606 & 8 & Mitochondria \\
\hline 16 & 284 & 0 & Peripheral neuron \\
\hline 13 & 183 & 9 & Tubule cell \\
\hline 12 & 1,855 & 5 & Macrophage \\
\hline 11 & 98 & 5 & Acinar cell \\
\hline 11 & 612 & 2 & Monocyte \\
\hline 10 & 18 & 0 & Thick ascending limb of loop of Henle \\
\hline 8 & 955 & 4 & Microglia \\
\hline 7 & 51 & 1 & Distal convoluted tubule \\
\hline 6 & 445 & 1 & Cytokine-mediated signaling pathway \\
\hline$\overline{6}$ & 1,534 & 0 & Endothelium (brain tissues only) \\
\hline 6 & 76 & 0 & Marginal zone B cell \\
\hline 5 & 139 & 0 & Ovarian vascular surface endothelium \\
\hline 5 & 339 & 1 & Sertoli cell \\
\hline 4 & 171 & 0 & Actin cytoskeleton \\
\hline 4 & 70 & 1 & AT2 cell \\
\hline$\overline{4}$ & 214 & 1 & Granulocyte \\
\hline 4 & 353 & 0 & Keratinocyte \\
\hline 4 & 52 & 1 & Parietal cell \\
\hline 3 & 1,843 & 0 & Dividing cell \\
\hline 3 & 41 & 0 & Intercalated cell of collecting duct \\
\hline 3 & 62 & 4 & Mast cell \\
\hline 3 & 118 & 3 & Megakaryocyte \\
\hline 3 & 3,802 & 1 & $\square$ Nucleolus \\
\hline 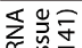 & 원 & 눈응 & Proportion of gene group \\
\hline$\underline{\underline{\alpha}}$ & प्र & 要党 & רחת \\
\hline ฮั है & 可 & ฮี่ & $\begin{array}{ccc}0 & 0.01 \geq 0.02 \\
\text { Module annotation category }\end{array}$ \\
\hline & 응 & 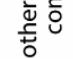 & $\begin{array}{l}\text { Pathway } \square \text { Cell compartment } 1 \text { Cell type } \\
\text { CNS cell } 1 \text { Immune cell }\end{array}$ \\
\hline
\end{tabular}

Fig. 5. Connecting genes through weighted gene co-expression network analysis (WGCNA). (A) Summary of gene assignment to modules by gene group and tissue. The underlying box plot shows the proportion of a gene group falling into that module status across tissues. Outlier point color indicates the tissue. (B) Proportion of lncRNA 
genes in modules across all tissues, binned by module size. Horizontal dashed lines indicate the highest and lowest proportions of lncRNA genes that met the expression threshold for WGCNA across all tissues $(0.29=$ testis, $0.20=$ whole blood). (C) Proportion of gene groups by intra-modular connectivity $\left(k_{i n}\right)$ ranking. The most-connected genes within their module are in the first $\mathrm{k}_{\text {in }}$ rank decile, and the least-connected genes within their module are in the tenth $\mathrm{k}_{\text {in }}$ rank decile. (D) Module annotations of genes with high intra-modular connectivity (the gene is in the top $\mathrm{k}_{\text {in }}$ rank decile of its module, and has scaled $k_{\text {in }} \geq 0.5$ ). Box fill reflects the proportion of genes assigned to a module with that annotation. Since genes are assigned to modules in multiple tissues, the labels reflect gene-tissue combinations, not individual genes. (E) Distribution of the number of modules shared between unique lncRNA/protein-coding gene pairs. (F) Location of the protein-coding gene MICA and the IncRNA gene AL645933.2, which share modules in 19 tissues and also are both outlier genes in 3 individuals. 
bioRxiv preprint doi: https://doi.org/10.1101/793091; this version posted October 4, 2019. The copyright holder for this preprint (which was not certified by peer review) is the author/funder, who has granted bioRxiv a license to display the preprint in perpetuity. It is made available under aCC-BY-NC 4.0 International license.

A

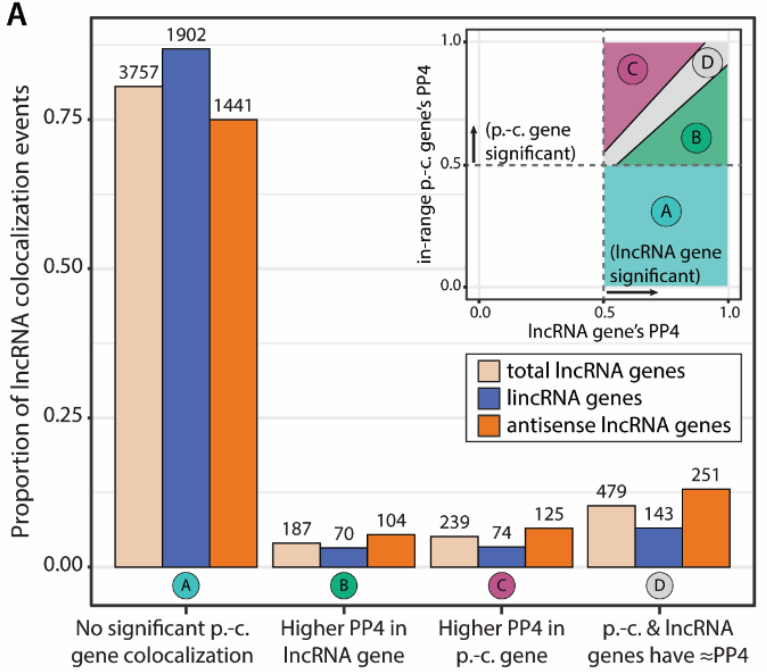

B

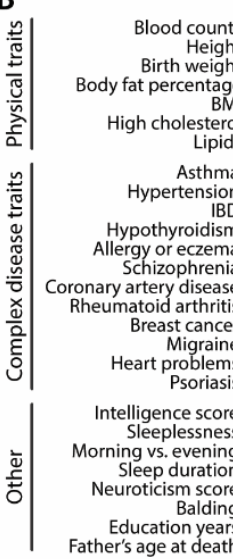

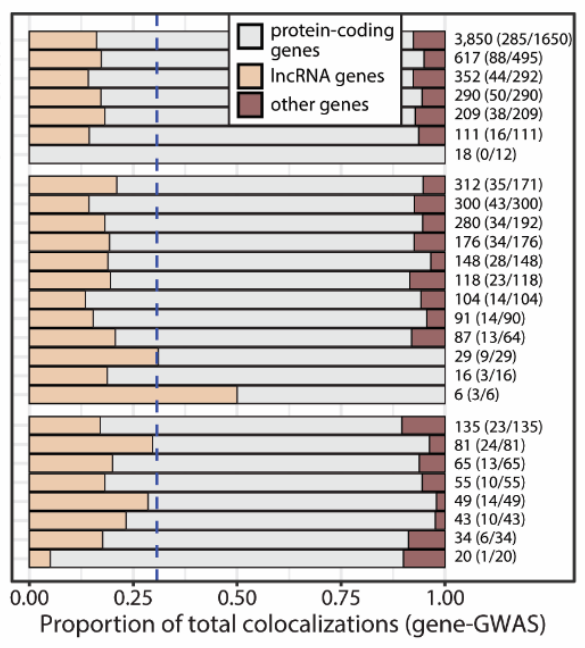
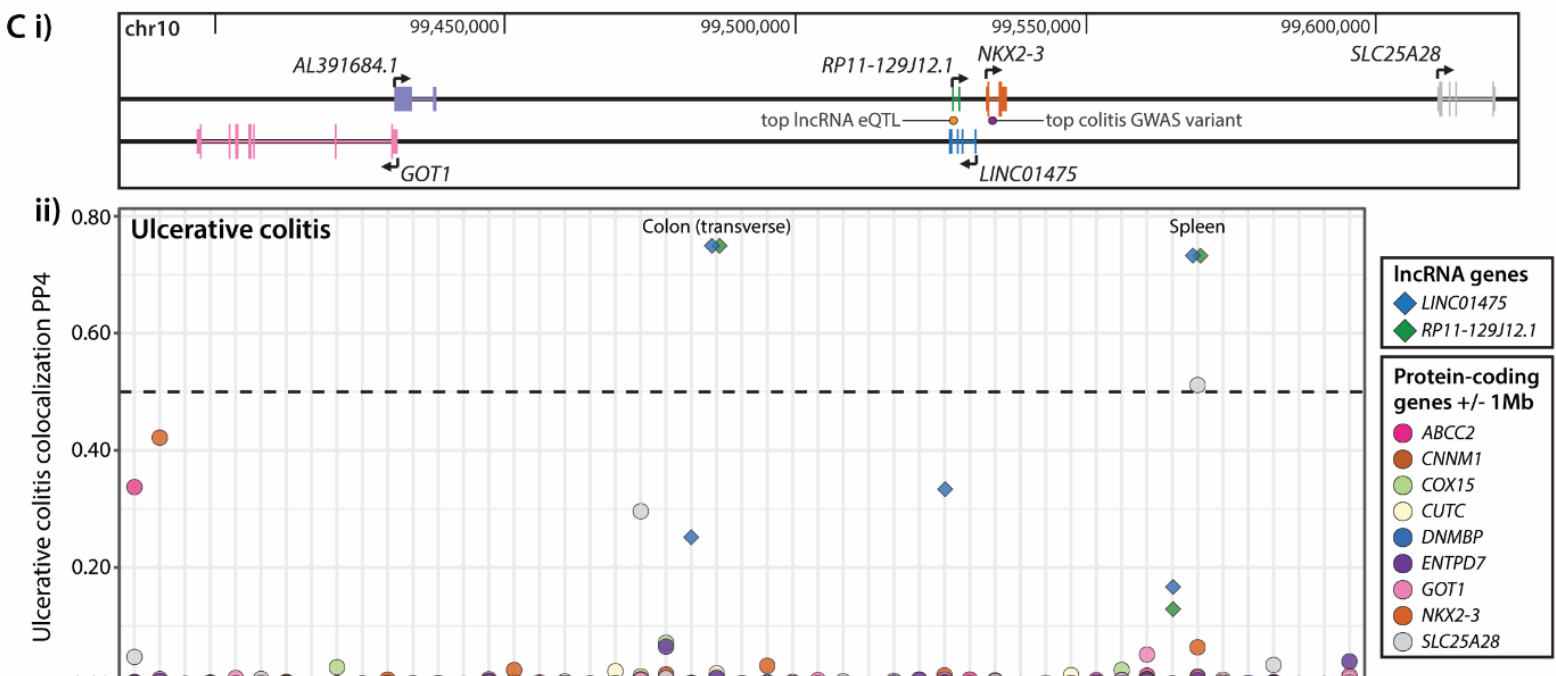

$0.00 \cdot 0000000080000008000800000800000000000008000008008$

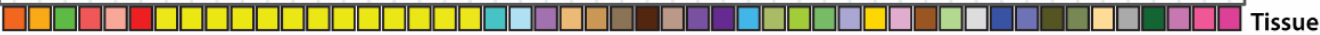
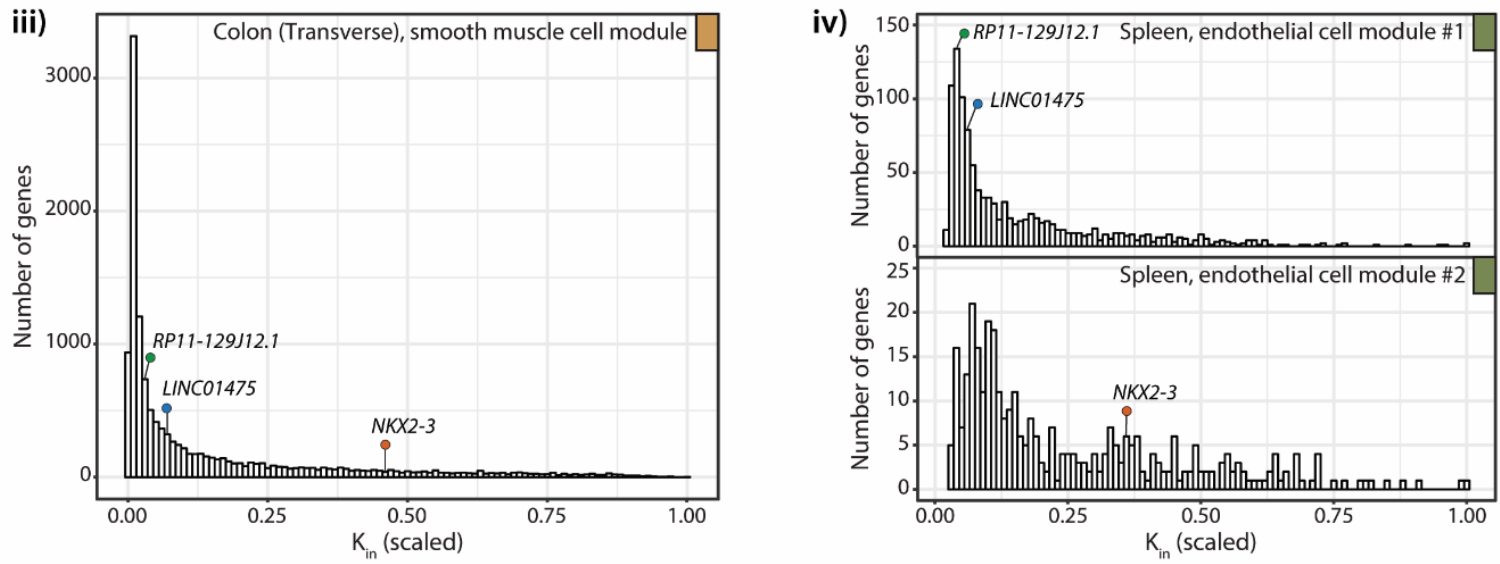

Fig. 6. GWAS-eQTL colocalization events involving lncRNA genes. (A) Summary of significant lncRNA colocalization events (event $=$ gene-tissue-GWAS combination) by their relationship to the protein-coding gene with the highest PP4 within $1 \mathrm{Mb}$ of the significant lncRNA gene. There were 32 significant IncRNA colocalization events where there was no protein-coding gene in range, which are not included in this plot. (B) Contribution of each gene type to significant colocalization events, collapsed across tissues (i.e. gene-GWAS combinations). 
GWASes were grouped on the y-axis by more general trait categories, and any traits with fewer than 5 significant gene-GWAS combinations were not included in this plot. The dashed line is the proportion of genes tested for colocalization that were IncRNAs (0.31). The numbers to the right of each bar show the total number of significant colocalization events (gene-trait-tissue combinations), followed by the number of unique significant lncRNA genes / the total number of unique significant genes in brackets. (C) Exemplar significant colocalizations with LINC01475 and RP11-129J12.1 and ulcerative colitis. i) Location of the lncRNA genes, as well as nearby protein-coding genes. Locations of the most significant ulcerative colitis GWAS variant and the top eQTL for both IncRNA genes in the transverse colon are indicated. ii) Colocalization posterior probability values across each tissue for LINCO1475 and RP11-129J12.1, as well as all protein-coding genes within 1Mb of the lncRNA genes, with ulcerative colitis. The dashed line indicates the threshold for significance, PP4 $\geq 0.5$. iii) Scaled intramodular connectivity $\left(\mathrm{k}_{\text {in }}\right)$ of

LINC01475, RP11-129J12.1, and NKX2-3 within their assigned smooth muscle cell module in the transverse colon gene co-expression network. iv) Scaled intramodular connectivity $\left(\mathrm{k}_{\text {in }}\right)$ of LINC01475, RP11-129J12.1, and NKX2-3 within their assigned endothelial cell modules (two separate modules) of the spleen gene co-expression network. 


\section{References and Notes:}

1. C.-C. Hon, J. A. Ramilowski, J. Harshbarger, N. Bertin, O. J. L. Rackham, J. Gough, E. Denisenko, S. Schmeier, T. M. Poulsen, J. Severin, M. Lizio, H. Kawaji, T. Kasukawa, M. Itoh, A. M. Burroughs, S. Noma, S. Djebali, T. Alam, Y. A. Medvedeva, A. C. Testa, L. Lipovich, C.-W. Yip, I. Abugessaisa, M. Mendez, A. Hasegawa, D. Tang, T. Lassmann, P. Heutink, M. Babina, C. A. Wells, S. Kojima, Y. Nakamura, H. Suzuki, C. O. Daub, M. J. L. de Hoon, E. Arner, Y. Hayashizaki, P. Carninci, A. R. R. Forrest, An atlas of human long non-coding RNAs with accurate 5' ends. Nature. 543, 199-204 (2017).

2. V. Amin, R. A. Harris, V. Onuchic, A. R. Jackson, T. Charnecki, S. Paithankar, S. Lakshmi Subramanian, K. Riehle, C. Coarfa, A. Milosavljevic, Epigenomic footprints across 111 reference epigenomes reveal tissue-specific epigenetic regulation of lincRNAs. Nat. Commun. 6, 6370 (2015).

3. M. Melé, K. Mattioli, W. Mallard, D. M. Shechner, C. Gerhardinger, J. L. Rinn, Chromatin environment, transcriptional regulation, and splicing distinguish lincRNAs and mRNAs. Genome Res. 27, 27-37 (2017).

4. J. J. Quinn, H. Y. Chang, Unique features of long non-coding RNA biogenesis and function. Nat. Rev. Genet. 17, 47-62 (2016).

5. S. Djebali, C. A. Davis, A. Merkel, A. Dobin, T. Lassmann, A. Mortazavi, A. Tanzer, J. Lagarde, W. Lin, F. Schlesinger, C. Xue, G. K. Marinov, J. Khatun, B. A. Williams, C. Zaleski, J. Rozowsky, M. Röder, F. Kokocinski, R. F. Abdelhamid, T. Alioto, I. Antoshechkin, M. T. Baer, N. S. Bar, P. Batut, K. Bell, I. Bell, S. Chakrabortty, X. Chen, J. Chrast, J. Curado, T. Derrien, J. Drenkow, E. Dumais, J. Dumais, R. Duttagupta, E. Falconnet, M. Fastuca, K. Fejes-Toth, P. Ferreira, S. Foissac, M. J. Fullwood, H. Gao, D. Gonzalez, A. Gordon, H. Gunawardena, C. Howald, S. Jha, R. Johnson, P. Kapranov, B. King, C. Kingswood, O. J. Luo, E. Park, K. Persaud, J. B. Preall, P. Ribeca, B. Risk, D. Robyr, M. Sammeth, L. Schaffer, L.-H. See, A. Shahab, J. Skancke, A. M. Suzuki, H. Takahashi, H. Tilgner, D. Trout, N. Walters, H. Wang, J. Wrobel, Y. Yu, X. Ruan, Y. Hayashizaki, J. Harrow, M. Gerstein, T. Hubbard, A. Reymond, S. E. Antonarakis, G. Hannon, M. C. Giddings, Y. Ruan, B. Wold, P. Carninci, R. Guigó, T. R. Gingeras, Landscape of transcription in human cells. Nature. 489, 101-108 (2012).

6. M. N. Cabili, C. Trapnell, L. Goff, M. Koziol, B. Tazon-Vega, A. Regev, J. L. Rinn, Integrative annotation of human large intergenic noncoding RNAs reveals global properties and specific subclasses. Genes Dev. 25, 1915-1927 (2011).

7. A. E. Kornienko, C. P. Dotter, P. M. Guenzl, H. Gisslinger, B. Gisslinger, C. Cleary, R. Kralovics, F. M. Pauler, D. P. Barlow, Long non-coding RNAs display higher natural expression variation than protein-coding genes in healthy humans. Genome Biol. 17, 14 (2016).

8. M. Melé, P. G. Ferreira, F. Reverter, D. S. DeLuca, J. Monlong, M. Sammeth, T. R. Young, J. M. Goldmann, D. D. Pervouchine, T. J. Sullivan, R. Johnson, A. V. Segrè, S. Djebali, A. Niarchou, The GTEx Consortium, F. A. Wright, T. Lappalainen, M. Calvo, G. Getz, E. T. Dermitzakis, K. G. Ardlie, R. Guigó, The human transcriptome across tissues and individuals. Science. 348, 660-665 (2015). 
9. K. C. Wang, H. Y. Chang, Molecular mechanisms of long noncoding RNAs. Mol. Cell. 43, 904-914 (2011).

10. M. K. Iyer, Y. S. Niknafs, R. Malik, U. Singhal, A. Sahu, Y. Hosono, T. R. Barrette, J. R. Prensner, J. R. Evans, S. Zhao, A. Poliakov, X. Cao, S. M. Dhanasekaran, Y.-M. Wu, D. R. Robinson, D. G. Beer, F. Y. Feng, H. K. Iyer, A. M. Chinnaiyan, The landscape of long noncoding RNAs in the human transcriptome. Nat. Genet. 47, 199-208 (2015).

11. S. Jiang, S.-J. Cheng, L.-C. Ren, Q. Wang, Y.-J. Kang, Y. Ding, M. Hou, X.-X. Yang, Y. Lin, N. Liang, G. Gao, An expanded landscape of human long noncoding RNA. Nucleic Acids Res. 10.1093/nar/gkz621 (2019).

12. P. J. Volders, K. Verheggen, G. Menschaert, K. Vandepoele, L. Martens, J. Vandesompele, P. Mestdagh, An update on LNCipedia: a database for annotated human lncRNA sequences. Nucleic Acids Res. 43, 4363-4364 (2015).

13. X. C. Quek, D. W. Thomson, J. L. V. Maag, N. Bartonicek, B. Signal, M. B. Clark, B. S. Gloss, M. E. Dinger, lncRNAdb v2.0: expanding the reference database for functional long noncoding RNAs. Nucleic Acids Research. 43, D168-D173 (2015).

14. Y. Liu, Z. Cao, Y. Wang, Y. Guo, P. Xu, P. Yuan, Z. Liu, Y. He, W. Wei, Genome-wide screening for functional long noncoding RNAs in human cells by Cas9 targeting of splice sites. Nat. Biotechnol. doi:10.1038/nbt.4283 (2018).

15. M. Sultan, V. Amstislavskiy, T. Risch, M. Schuette, S. Dökel, M. Ralser, D. Balzereit, H. Lehrach, M.-L. Yaspo, Influence of RNA extraction methods and library selection schemes on RNA-seq data. BMC Genomics. 15, 675 (2014).

16. T. Derrien, R. Johnson, G. Bussotti, A. Tanzer, S. Djebali, H. Tilgner, G. Guernec, D. Martin, A. Merkel, D. G. Knowles, J. Lagarde, L. Veeravalli, X. Ruan, Y. Ruan, T. Lassmann, P. Carninci, J. B. Brown, L. Lipovich, J. M. Gonzalez, M. Thomas, C. A. Davis, R. Shiekhattar, T. R. Gingeras, T. J. Hubbard, C. Notredame, J. Harrow, R. Guigó, The GENCODE v7 catalog of human long noncoding RNAs: analysis of their gene structure, evolution, and expression. Genome Res. 22, 1775-1789 (2012).

17. B. Xia, M. Baron, Y. Yan, F. Wagner, S. Y. Kim, D. L. Keefe, J. P. Alukal, J. D. Boeke, I. Yanai, Widespread transcriptional scanning in the testis modulates gene evolution rates. https://www.biorxiv.org/content/10.1101/282129v2 (2018).

18. GTEx Consortium, The GTEx Consortium atlas of genetic regulatory effects across human tissues. BioRxiv. (2019).

19. K. Mattioli, P.-J. Volders, C. Gerhardinger, J. C. Lee, P. G. Maass, M. Melé, J. L. Rinn, High-throughput functional analysis of lncRNA core promoters elucidates rules governing tissue specificity. Genome Res. 29, 344-355 (2019).

20. P. Mohammadi, S. E. Castel, A. A. Brown, T. Lappalainen, Quantifying the regulatory effect size of cis-acting genetic variation using allelic fold change. Genome Res. 27, 1872-1884 (2017).

21. K. Popadin, M. Gutierrez-Arcelus, E. T. Dermitzakis, S. E. Antonarakis, Genetic and epigenetic regulation of human lincRNA gene expression. Am. J. Hum. Genet. 93, 1015-1026 (2013). 
22. J. Lagarde, B. Uszczynska-Ratajczak, S. Carbonell, S. Pérez-Lluch, A. Abad, C. Davis, T. R. Gingeras, A. Frankish, J. Harrow, R. Guigo, R. Johnson, High-throughput annotation of fulllength long noncoding RNAs with capture long-read sequencing. Nat. Genet. 49, 1731-1740 (2017).

23. N. M. Ferraro, B. J. Strober, J. Einson, X. Li, F. Aguet, A. N. Barbeira, S. E. Castel, J. R. Davis, A. Hilliard, B. Kotis, Y. Park, A. J. Scott, C. Smail, E. K. Tsang, K. G. Ardlie, T. Assimes, I. Hall, H. K. Im, GTEx Consortium, T. Lappalainen, P. Mohammadi, S. B. Montgomery, A. Battle, Diverse transcriptomic signatures across human tissues identify functional rare genetic variation. BioRxiv. (2019).

24. S. Guil, M. Esteller, Cis-acting noncoding RNAs: friends and foes. Nat. Struct. Mol. Biol. 19, 1068-1075 (2012).

25. A. Saha, A. Battle, False positives in trans-eQTL and co-expression analyses arising from RNA-sequencing alignment errors. F1000Res. 7, 1860 (2018).

26. C. J. Brown, A. Ballabio, J. L. Rupert, R. G. Lafreniere, M. Grompe, R. Tonlorenzi, H. F. Willard, A gene from the region of the human $\mathrm{X}$ inactivation centre is expressed exclusively from the inactive X chromosome. Nature. 349, 38-44 (1991).

27. P. Avner, E. Heard, X-chromosome inactivation: counting, choice and initiation. Nat. Rev. Genet. 2, 59-67 (2001).

28. P. Langfelder, S. Horvath, WGCNA: an R package for weighted correlation network analysis. BMC Bioinformatics. 9, 559 (2008).

29. X. Han, R. Wang, Y. Zhou, L. Fei, H. Sun, S. Lai, A. Saadatpour, Z. Zhou, H. Chen, F. Ye, D. Huang, Y. Xu, W. Huang, M. Jiang, X. Jiang, J. Mao, Y. Chen, C. Lu, J. Xie, Q. Fang, Y. Wang, R. Yue, T. Li, H. Huang, S. H. Orkin, G.-C. Yuan, M. Chen, G. Guo, Mapping the Mouse Cell Atlas by Microwell-Seq. Cell. 173, 1307 (2018).

30. V. Groh, R. Rhinehart, J. Randolph-Habecker, M. S. Topp, S. R. Riddell, T. Spies, Costimulation of CD8 $\alpha \beta$ T cells by NKG2D via engagement by MIC induced on virusinfected cells. Nat. Immunol. 2, 255-260 (2001).

31. H. Das, V. Groh, C. Kuijl, M. Sugita, C. T. Morita, T. Spies, J. F. Bukowski, MICA Engagement by Human V 22 V 2 T Cells Enhances Their Antigen-Dependent Effector Function. Immunity. 15, 83-93 (2001).

32. J. Zhang, F. Basher, J. D. Wu, NKG2D Ligands in Tumor Immunity: Two Sides of a Coin. Frontiers in Immunology. 6, 97 (2015).

33. GTEx GWAS Subgroup, Downstream consequences of genetic regulatory effects on complex human disease. BioRxiv. (2019).

34. X. Lu, L. Tang, K. Li, J. Zheng, P. Zhao, Y. Tao, L.-X. Li, Contribution of NKX2-3 Polymorphisms to Inflammatory Bowel Diseases: A Meta-Analysis of 35358 subjects. Scientific Reports. 4, 3924 (2015).

35. O. Pabst, R. Zweigerdt, H. H. Arnold, Targeted disruption of the homeobox transcription factor Nkx2-3 in mice results in postnatal lethality and abnormal development of small intestine and spleen. Development. 126, 2215-2225 (1999). 
36. O. Pabst, R. Förster, M. Lipp, H. Engel, H. H. Arnold, NKX2.3 is required for MAdCAM-1 expression and homing of lymphocytes in spleen and mucosa-associated lymphoid tissue. EMBO J. 19, 2015-2023 (2000).

37. E. F. Robles, M. Mena-Varas, L. Barrio, S. V. Merino-Cortes, P. Balogh, M.-Q. Du, T. Akasaka, A. Parker, S. Roa, C. Panizo, I. Martin-Guerrero, R. Siebert, V. Segura, X. Agirre, L. Macri-Pellizeri, B. Aldaz, A. Vilas-Zornoza, S. Zhang, S. Moody, M. J. Calasanz, T. Tousseyn, C. Broccardo, P. Brousset, E. Campos-Sanchez, C. Cobaleda, I. Sanchez-Garcia, J. L. Fernandez-Luna, R. Garcia-Muñoz, E. Pena, B. Bellosillo, A. Salar, M. J. Baptista, J. M. Hernandez-Rivas, M. Gonzalez, M. J. Terol, J. Climent, A. Ferrandez, X. Sagaert, A. M. Melnick, F. Prosper, D. G. Oscier, Y. R. Carrasco, M. J. S. Dyer, J. A. Martinez-Climent, Homeobox NKX2-3 promotes marginal-zone lymphomagenesis by activating B-cell receptor signalling and shaping lymphocyte dynamics. Nature Communications. 7, 11889 (2016).

38. D. Tarlinton, A. Light, D. Metcalf, R. P. Harvey, L. Robb, Architectural Defects in the Spleens of Nkx2-3-Deficient Mice Are Intrinsic and Associated with Defects in Both B Cell Maturation and T Cell-Dependent Immune Responses. The Journal of Immunology. 170, 4002-4010 (2003).

39. D. Vojkovics, Z. Kellermayer, B. Kajtár, G. Roncador, Á. Vincze, P. Balogh, Nkx2-3-A Slippery Slope From Development Through Inflammation Toward Hematopoietic Malignancies. Biomarker Insights. 13, 117727191875748 (2018).

40. J. Carlevaro-Fita, L. Liu, Y. Zhou, S. Zhang, P. Chouvardas, R. Johnson, J. Li, LnCompare: gene set feature analysis for human long non-coding RNAs. Nucleic Acids Research. 47, W523-W529 (2019).

41. U. Perron, P. Provero, I. Molineris, In silico prediction of lncRNA function using tissue specific and evolutionary conserved expression. BMC Bioinformatics. 18, 144 (2017).

42. S. C. Pyfrom, H. Luo, J. E. Payton, PLAIDOH: a novel method for functional prediction of long non-coding RNAs identifies cancer-specific LncRNA activities. BMC Genomics. 20, 137 (2019).

43. J. Zhou, Y. Huang, Y. Ding, J. Yuan, H. Wang, H. Sun, lncFunTK: a toolkit for functional annotation of long noncoding RNAs. Bioinformatics. 34, 3415-3416 (2018).

44. D. S. DeLuca, J. Z. Levin, A. Sivachenko, T. Fennell, M.-D. Nazaire, C. Williams, M. Reich, W. Winckler, G. Getz, RNA-SeQC: RNA-seq metrics for quality control and process optimization. Bioinformatics. 28, 1530-1532 (2012).

45. B. B. Hansen, S. O. Klopfer, Optimal Full Matching and Related Designs via Network Flows. Journal of Computational and Graphical Statistics. 15, 609-627 (2006).

46. S. M. Urbut, G. Wang, P. Carbonetto, M. Stephens, Flexible statistical methods for estimating and testing effects in genomic studies with multiple conditions. Nat. Genet. 51, 187-195 (2019).

47. O. Stegle, L. Parts, M. Piipari, J. Winn, R. Durbin, Using probabilistic estimation of expression residuals (PEER) to obtain increased power and interpretability of gene expression analyses. Nat. Protoc. 7, 500-507 (2012). 
48. C. Chiang, A. J. Scott, J. R. Davis, E. K. Tsang, X. Li, Y. Kim, T. Hadzic, F. N. Damani, L. Ganel, GTEx Consortium, S. B. Montgomery, A. Battle, D. F. Conrad, I. M. Hall, The impact of structural variation on human gene expression. Nat. Genet. 49, 692-699 (2017).

49. R. E. Handsaker, V. Van Doren, J. R. Berman, G. Genovese, S. Kashin, L. M. Boettger, S. A. McCarroll, Large multiallelic copy number variations in humans. Nat. Genet. 47, 296-303 (2015).

50. E. J. Gardner, V. K. Lam, D. N. Harris, N. T. Chuang, E. C. Scott, W. S. Pittard, R. E. Mills, 1000 Genomes Project Consortium, S. E. Devine, The Mobile Element Locator Tool (MELT): population-scale mobile element discovery and biology. Genome Res. 27, 19161929 (2017).

51. K. J. Karczewski, L. C. Francioli, G. Tiao, B. B. Cummings, Variation across 141,456 human exomes and genomes reveals the spectrum of loss-of-function intolerance across human protein-coding genes. https://www.biorxiv.org/content/10.1101/531210v3 (2019).

52. S. E. Castel, A. Levy-Moonshine, P. Mohammadi, E. Banks, T. Lappalainen, Tools and best practices for data processing in allelic expression analysis. Genome Biology. 16, 195 (2015).

53. B. van de Geijn, G. McVicker, Y. Gilad, J. K. Pritchard, WASP: allele-specific software for robust molecular quantitative trait locus discovery. Nat. Methods. 12, 1061-1063 (2015).

54. S. E. Castel, F. Aguet, P. Mohammadi, GTEx Consortium, K. G. Ardlie, T. Lappalainen, A vast resource of allelic expression data spanning human tissues. BioRxiv. (2019).

55. N. I. Panousis, M. Gutierrez-Arcelus, E. T. Dermitzakis, T. Lappalainen, Allelic mapping bias in RNA-sequencing is not a major confounder in eQTL studies. Genome Biol. 15, 467 (2014).

56. W. Huber, A. von Heydebreck, H. Sultmann, A. Poustka, M. Vingron, Variance stabilization applied to microarray data calibration and to the quantification of differential expression. Bioinformatics. 18, S96-S104 (2002).

57. P. Langfelder, B. Zhang, S. Horvath, Defining clusters from a hierarchical cluster tree: the Dynamic Tree Cut package for R. Bioinformatics. 24, 719-720 (2008).

58. M.Ashburner, C. A. Ball, J. A. Blake, D. Botstein, H. Butler, J. Michael Cherry, A. P. Davis, K. Dolinski, S. S. Dwight, J. T. Eppig, M. A. Harris, D. P. Hill, L. Issel-Tarver, A. Kasarskis, S. Lewis, J. C. Matese, J. E. Richardson, M. Ringwald, G. M. Rubin, G. Sherlock, Gene Ontology: tool for the unification of biology. Nature Genetics. 25, 25-29 (2000).

59. The Gene Ontology Consortium, The Gene Ontology Consortium, The Gene Ontology Resource: 20 years and still GOing strong. Nucleic Acids Research. 47, D330-D338 (2019)

60. J. D. Dougherty, E. F. Schmidt, M. Nakajima, N. Heintz, Analytical approaches to RNA profiling data for the identification of genes enriched in specific cells. Nucleic Acids Research. 38, 4218-4230 (2010).

61. X. Xu, A. B. Wells, D. R. O’Brien, A. Nehorai, J. D. Dougherty, Cell Type-Specific Expression Analysis to Identify Putative Cellular Mechanisms for Neurogenetic Disorders. Journal of Neuroscience. 34, 1420-1431 (2014). 
62. E. Y. Chen, C. M. Tan, Y. Kou, Q. Duan, Z. Wang, G. Meirelles, N. R. Clark, A. Ma'ayan, Enrichr: interactive and collaborative HTML5 gene list enrichment analysis tool. $B M C$ Bioinformatics. 14, 128 (2013).

63. M. V. Kuleshov, M. R. Jones, A. D. Rouillard, N. F. Fernandez, Q. Duan, Z. Wang, S. Koplev, S. L. Jenkins, K. M. Jagodnik, A. Lachmann, M. G. McDermott, C. D. Monteiro, G. W. Gundersen, A. Ma'ayan, Enrichr: a comprehensive gene set enrichment analysis web server 2016 update. Nucleic Acids Res. 44, W90-7 (2016).

64. N. Novershtern, A. Subramanian, L. N. Lawton, R. H. Mak, W. Nicholas Haining, M. E. McConkey, N. Habib, N. Yosef, C. Y. Chang, T. Shay, G. M. Frampton, A. C. B. Drake, I. Leskov, B. Nilsson, F. Preffer, D. Dombkowski, J. W. Evans, T. Liefeld, J. S. Smutko, J. Chen, N. Friedman, R. A. Young, T. R. Golub, A. Regev, B. L. Ebert, Densely Interconnected Transcriptional Circuits Control Cell States in Human Hematopoiesis. Cell. 144, 296-309 (2011).

65. L. Gautier, L. Cope, B. M. Bolstad, R. A. Irizarry, affy--analysis of Affymetrix GeneChip data at the probe level. Bioinformatics. 20, 307-315 (2004).

66. S. Darmanis, S. A. Sloan, Y. Zhang, M. Enge, C. Caneda, L. M. Shuer, M. G. Hayden Gephart, B. A. Barres, S. R. Quake, A survey of human brain transcriptome diversity at the single cell level. Proceedings of the National Academy of Sciences. 112, 7285-7290 (2015). 


\section{Acknowledgments:}

We thank the Montgomery and Kirkegaard labs for their feedback on this work.

Funding: The GTEx Project was supported by the Common Fund of the Office of the Director of the National Institutes of Health (NIH) and by the National Cancer Institute (NCI), the National Human Genome Research Institute (NHGRI), the National Heart, Lung, and Blood Institute (NHLBI), the National Institute on Drug Abuse (NIDA), the National Institute of Mental Health (NIMH) and the National Institute of Neurological Disorders and Stroke (NINDS). We are thankful for support from a Gabilan Stanford Graduate Fellowship (O.M.de G.), a Bio-X Stanford Interdisciplinary Graduate Fellowship (O.M.de G.), National Science Foundation Graduate Research Fellowship (N.M.F.), NHLBI grant R01HL135313-01 (A.S.R.), grants HHSN268201000029C and 5U41HG009494 (F.A., K.G.A.), NIH grant R01GM122924 (S.E.C., T.L.), grant 1K99HG009916-01 (S.E.C.), a Marie-Skłodowska Curie fellowship H2020 Grant 706636 (S.K.-H.), NIH grant R01HG010067 (Y.P.), a Mr. and Mrs. Spencer T. Olin Fellowship for Women in Graduate Study (A.J.S.), grant R01MH109905 (A.B.), the Searle Scholar Program (A.B.), grant R01MH101822 (C.D.B.), NIH grants R01MH106842, R01HL142028, UM1HG008901, and R01GM124486 (T.L.), NIH grants R01MH107666 and P30DK020595 (H.K.I.), NIH grants R01HL109512, R01HL134817, R33HL120757, and R01HL139478 (T.Q.), the Chan Zuckerberg Foundation - Human Cell Atlas Initiative (T.Q.), Stanford University School of Medicine (K.K.), NIH grants R01MH101814 (NIH Common Fund; GTEx Program) (A.B., S.B.M), R01HG008150 (NHGRI; Non-Coding Variants Program) (A.B., S.B.M), R01HL142015, U01HG009431, and U01HG009080 (S.B.M).

Author contributions: O.M.de G. co-led manuscript, conducted analyses, visualized data, and co-wrote manuscript; N.M.F. conducted outlier analysis and contributed to writing; D.C.N. conducted network analysis; A.S.R. contributed to colocalization analysis; F.A. generated QTL and ASE data, and provided feedback on manuscript; A.N.B. contributed to GWAS harmonization and colocalization analysis; S.E.C. generated ASE and tissue sharing (MashR) data and provided feedback on manuscript; S.K.-H. generated tissue sharing (MashR) data and provided feedback on manuscript; Y.P. contributed to colocalization analysis and provided feedback on manuscript; A.J.S. generated structural variant data and provided feedback on manuscript; B.J.S. contributed to outlier analysis; A.B. contributed to outlier analysis and provided feedback on manuscript; C.D.B. led trainees and contributed to GWAS analysis; X.W. led trainees and contributed to colocalization analysis; I.M.H. led trainees and contributed to structural variant data; T.L. led trainees and provided feedback on manuscript; H.K.I. led trainees and led the GWAS analysis team; K.G.A. generated data, provided oversight of LDACC and pipelines, and provided feedback on manuscript; T.Q. helped with data interpretation and provided feedback on manuscript; K.K. helped with data interpretation and provided feedback on manuscript; S.B.M. co-led manuscript, led trainees, and co-wrote manuscript.

Competing interests: F.A. is an inventor on a patent application related to TensorQTL; S.E.C. is a co-founder and chief technology officer at Variant Bio, and owns stock in Variant Bio; T.L. is on the scientific advisory board of Variant Bio and Goldfinch Bio, and owns stock in Variant Bio; S.B.M. is on the scientific advisory board of Prime Genomics Inc.

Data and materials availability: All data used for these analyses are available through dbGaP (accession phs000424.v8) and the GTEx Portal (www.gtexportal.org). 


\section{Supplementary Materials:}

Materials and Methods

Figures S1-S9

Tables S1-S8

References (44-66)

\section{GTEx Consortium Information:}

Laboratory and Data Analysis Coordinating Center (LDACC): François Aguet ${ }^{1}$, Shankara Anand $^{1}$, Kristin G Ardlie ${ }^{1}$, Stacey Gabriel ${ }^{1}$, Gad Getz ${ }^{1,2}$, Aaron Graubert ${ }^{1}$, Kane Hadley ${ }^{1}$, Robert E Handsaker $^{3,4,5}$, Katherine H Huang ${ }^{1}$, Seva Kashin ${ }^{3,4,5}$, Xiao Li ${ }^{1}$, Daniel G MacArthur ${ }^{4,6}$, Samuel R Meier ${ }^{1}$, Jared L Nedzel ${ }^{1}$, Duyen Y Nguyen ${ }^{1}$, Ayellet V Segrè ${ }^{1,7}$, Ellen Todres ${ }^{1}$

Analysis Working Group (funded by GTEx project grants): François Aguet ${ }^{1}$, Shankara Anand $^{1}$, Kristin G Ardlie ${ }^{1}$, Brunilda Balliu ${ }^{8}$, Alvaro N Barbeira ${ }^{9}$, Alexis Battle ${ }^{10,11}$, Rodrigo Bonazzola $^{9}$, Andrew Brown ${ }^{12,13}$, Christopher D Brown ${ }^{14}$, Stephane E Castel ${ }^{15,16}$, Don Conrad $^{17,18}$, Daniel J Cotter ${ }^{19}$, Nancy Cox ${ }^{20}$, Sayantan Das ${ }^{21}$, Olivia M de Goede ${ }^{19}$, Emmanouil T Dermitzakis $^{12,22,23}$, Barbara E Engelhardt ${ }^{24,25}$, Eleazar Eskin ${ }^{26}$, Tiffany Y Eulalio ${ }^{27}$, Nicole M Ferraro $^{27}$, Elise Flynn ${ }^{15,16}$, Laure Fresard ${ }^{28}$, Eric R Gamazon ${ }^{29,30,31,20}$, Diego Garrido-Martín ${ }^{32}$, Nicole R Gay ${ }^{19}$, Gad Getz ${ }^{1,2}$, Aaron Graubert ${ }^{1}$, Roderic Guigó ${ }^{32,33}$, Kane Hadley ${ }^{1}$, Andrew R Hamel $^{7,1}$, Robert E Handsaker ${ }^{3,4,5}$, Yuan He ${ }^{10}$, Paul J Hoffman ${ }^{15}$, Farhad Hormozdiari ${ }^{34,1}$, Lei Hou $^{35,1}$, Katherine H Huang ${ }^{1}$, Hae Kyung Im ${ }^{9}$, Brian Jo ${ }^{24,25}$, Silva Kasela ${ }^{15,16}$, Seva Kashin ${ }^{3,4,5}$, Manolis Kellis ${ }^{35,1}$, Sarah Kim-Hellmuth ${ }^{15,16,36}$, Alan Kwong ${ }^{21}$, Tuuli Lappalainen ${ }^{15,16}$, Xiao Li ${ }^{1}$, Xin Li $^{28}$, Yanyu Liang 9 , Daniel G MacArthur ${ }^{4,6}$, Serghei Mangul ${ }^{26,37}$, Samuel R Meier ${ }^{1}$, Pejman Mohammadi $^{15,16,38,39}$, Stephen B Montgomery ${ }^{28,19}$, Manuel Muñoz-Aguirre ${ }^{32,40}$, Daniel C Nachun $^{28}$, Jared L Nedzel ${ }^{1}$, Duyen Y Nguyen ${ }^{1}$, Andrew B Nobel ${ }^{41}$, Meritxell Oliva ${ }^{9,42}$, YoSon Park $^{14,43}$, Yongjin Park ${ }^{35,1}$, Princy Parsana ${ }^{11}$, Ferran Reverter ${ }^{44}$, John M Rouhana ${ }^{7,1}$, Chiara Sabatti $^{45}$, Ashis Saha ${ }^{11}$, Ayellet V Segrè ${ }^{1,7}$, Andrew D Skol ${ }^{9,46}$, Matthew Stephens ${ }^{47}$, Barbara E Stranger ${ }^{9,48}$, Benjamin J Strober ${ }^{10}$, Nicole A Teran ${ }^{28}$, Ellen Todres ${ }^{1}$, Ana Viñuela ${ }^{49,12,22,23}$, Gao Wang $^{47}$, Xiaoquan Wen ${ }^{21}$, Fred Wright ${ }^{50}$, Valentin Wucher ${ }^{32}$, Yuxin Zou ${ }^{51}$

Analysis Working Group (not funded by GTEx project grants): Pedro G Ferreira ${ }^{52,53,54}$, Gen Li $^{55}$, Marta Melé ${ }^{56}$, Esti Yeger-Lotem ${ }^{57,58}$

Leidos Biomedical - Project Management: Mary E Barcus ${ }^{59}$, Debra Bradbury ${ }^{60}$, Tanya Krubit $^{60}$, Jeffrey A McLean ${ }^{60}$, Liqun Qi ${ }^{60}$, Karna Robinson ${ }^{60}$, Nancy V Roche ${ }^{60}$, Anna M Smith $^{60}$, Leslie Sobin ${ }^{60}$, David E Tabor ${ }^{60}$, Anita Undale ${ }^{60}$

Biospecimen collection source sites: Jason Bridge ${ }^{61}$, Lori E Brigham ${ }^{62}$, Barbara A Foster ${ }^{63}$, Bryan M Gillard ${ }^{63}$, Richard Hasz ${ }^{64}$, Marcus Hunter ${ }^{65}$, Christopher Johns ${ }^{66}$, Mark Johnson ${ }^{67}$, Ellen Karasik $^{63}$, Gene Kopen ${ }^{68}$, William F Leinweber ${ }^{68}$, Alisa McDonald ${ }^{68}$, Michael T Moser ${ }^{63}$, Kevin Myer $^{65}$, Kimberley D Ramsey ${ }^{63}$, Brian Roe ${ }^{65}$, Saboor Shad ${ }^{68}$, Jeffrey A Thomas ${ }^{68,67}$, Gary Walters $^{67}$, Michael Washington ${ }^{67}$, Joseph Wheeler ${ }^{66}$

Brain bank repository: David A Davis ${ }^{70}$, Deborah C Mash ${ }^{70}$ 
Pathology: Mary E Barcus ${ }^{59}$, Philip A Branton ${ }^{71}$, Leslie Sobin ${ }^{60}$

ELSI study: Laura K Barker ${ }^{72}$, Heather M Gardiner ${ }^{72}$, Maghboeba Mosavel ${ }^{73}$, Laura A Siminoff ${ }^{72}$

Genome Browser Data Integration \& Visualization: Paul Flicek ${ }^{74}$, Maximilian Haeussler ${ }^{75}$, Thomas Juettemann $^{74}$, W James Kent ${ }^{75}$, Christopher M Lee ${ }^{75}$, Conner C Powell ${ }^{75}$, Kate R Rosenbloom $^{75}$, Magali Ruffier ${ }^{74}$, Dan Sheppard ${ }^{74}$, Kieron Taylor ${ }^{74}$, Stephen J Trevanion ${ }^{74}$, Daniel R Zerbino ${ }^{74}$

eGTEx groups: Nathan S Abell ${ }^{19}$, Joshua Akey ${ }^{76}$, Lin Chen ${ }^{42}$, Kathryn Demanelis ${ }^{42}$, Jennifer A Doherty $^{77}$, Andrew P Feinberg ${ }^{78}$, Kasper D Hansen ${ }^{79}$, Peter F Hickey ${ }^{80}$, Lei Hou ${ }^{35,1}$, Farzana Jasmine $^{42}$, Lihua Jiang ${ }^{19}$, Rajinder Kaul ${ }^{81,82}$, Manolis Kellis ${ }^{35,1}$, Muhammad G Kibriya ${ }^{42}$, Jin Billy Li ${ }^{19}$, Qin Li ${ }^{19}$, Shin Lin ${ }^{83}$, Sandra E Linder ${ }^{19}$, Stephen B Montgomery ${ }^{28,19}$, Meritxell Oliva $^{9,42}$, Yongjin Park ${ }^{35,1}$, Brandon L Pierce ${ }^{42}$, Lindsay F Rizzardi ${ }^{84}$, Andrew D Skol ${ }^{9,46}$, Kevin S Smith $^{28}$, Michael Snyder ${ }^{19}$, John Stamatoyannopoulos ${ }^{81,85}$, Barbara E Stranger ${ }^{9,48}$, Hua Tang ${ }^{19}$, Meng Wang ${ }^{19}$

NIH program management: Philip A Branton ${ }^{71}$, Latarsha J Carithers ${ }^{71,86}$, Ping Guan ${ }^{71}$, Susan E Koester $^{87}$, A. Roger Little ${ }^{88}$, Helen M Moore ${ }^{71}$, Concepcion R Nierras ${ }^{89}$, Abhi K Rao ${ }^{71}$, Jimmie B Vaught $^{71}$, Simona Volpi ${ }^{90}$

\section{Affiliations}

1. The Broad Institute of MIT and Harvard, Cambridge, MA, USA

2. Cancer Center and Department of Pathology, Massachusetts General Hospital, Boston, MA, USA

3. Department of Genetics, Harvard Medical School, Boston, MA, USA

4. Program in Medical and Population Genetics, The Broad Institute of Massachusetts Institute of Technology and Harvard University, Cambridge, MA, USA

5. Stanley Center for Psychiatric Research, Broad Institute, Cambridge, MA, USA

6. Analytic and Translational Genetics Unit, Massachusetts General Hospital, Boston, MA, USA

7. Ocular Genomics Institute, Massachusetts Eye and Ear, Harvard Medical School, Boston, MA, USA

8. Department of Biomathematics, University of California, Los Angeles, Los Angeles, CA, USA

9. Section of Genetic Medicine, Department of Medicine, The University of Chicago, Chicago,

IL, USA

10. Department of Biomedical Engineering, Johns Hopkins University, Baltimore, MD, USA

11. Department of Computer Science, Johns Hopkins University, Baltimore, MD, USA

12. Department of Genetic Medicine and Development, University of Geneva Medical School, Geneva, Switzerland

13. Population Health and Genomics, University of Dundee, Dundee, Scotland, UK

14. Department of Genetics, University of Pennsylvania, Perelman School of Medicine, 
17. Department of Genetics, Washington University School of Medicine, St. Louis, Missouri, USA

18. Department of Pathology \& Immunology, Washington University School of Medicine, St. Louis, Missouri, USA

19. Department of Genetics, Stanford University, Stanford, CA, USA

20. Division of Genetic Medicine, Department of Medicine, Vanderbilt University Medical Center, Nashville, TN, USA

21. Department of Biostatistics, University of Michigan, Ann Arbor, MI, USA

22. Institute for Genetics and Genomics in Geneva (iGE3), University of Geneva, Geneva,

23. Swiss Institute of Bioinformatics, Geneva, Switzerland

24. Department of Computer Science, Princeton University, Princeton, NJ, USA

25. Center for Statistics and Machine Learning, Princeton University, Princeton, NJ, USA

26. Department of Computer Science, University of California, Los Angeles, Los Angeles, CA, USA

27. Program in Biomedical Informatics, Stanford University School of Medicine, Stanford, CA, USA

28. Department of Pathology, Stanford University, Stanford, CA, USA

29. Data Science Institute, Vanderbilt University, Nashville, TN, USA

30. Clare Hall, University of Cambridge, Cambridge, UK

31. MRC Epidemiology Unit, University of Cambridge, Cambridge, UK

32. Centre for Genomic Regulation (CRG), The Barcelona Institute for Science and Technology, Barcelona, Catalonia, Spain

33. Universitat Pompeu Fabra (UPF), Barcelona, Catalonia, Spain

34. Department of Epidemiology, Harvard T.H. Chan School of Public Health, Boston, MA, USA

35. Computer Science and Artificial Intelligence Laboratory, Massachusetts Institute of

Technology, Cambridge, MA, USA

36. Statistical Genetics, Max Planck Institute of Psychiatry, Munich, Germany

37. Department of Clinical Pharmacy, School of Pharmacy, University of Southern California, Los Angeles, CA, USA

38. Scripps Research Translational Institute, La Jolla, CA, USA

39. Department of Integrative Structural and Computational Biology, The Scripps Research Institute, La Jolla, CA, USA

40. Department of Statistics and Operations Research, Universitat Politècnica de Catalunya (UPC), Barcelona, Catalonia, Spain

41. Department of Statistics and Operations Research and Department of Biostatistics, University of North Carolina, Chapel Hill, NC, USA

42. Department of Public Health Sciences, The University of Chicago, Chicago, IL, USA

43. Department of Systems Pharmacology and Translational Therapeutics, University of Pennsylvania, Perelman School of Medicine, Philadelphia, PA, USA

44. Department of Genetics, Microbiology and Statistics, University of Barcelona, Barcelona. Spain.

45. Departments of Biomedical Data Science and Statistics, Stanford University, Stanford, CA, USA 
46. Department of Pathology and Laboratory Medicine, Ann \& Robert H. Lurie Children's Hospital of Chicago, Chicago, IL, USA

47. Department of Human Genetics, University of Chicago, Chicago, IL, USA

48. Center for Genetic Medicine, Department of Pharmacology, Northwestern University,

49. Department of Twin Research and Genetic Epidemiology, King's College London, London, UK

50. Bioinformatics Research Center and Departments of Statistics and Biological Sciences, North Carolina State University, Raleigh, NC, USA

51. Department of Statistics, University of Chicago, Chicago, IL, USA

52. Department of Computer Sciences, Faculty of Sciences, University of Porto, Porto, Portugal

53. Instituto de Investigação e Inovação em Saúde, Universidade do Porto, Porto, Portugal

54. Institute of Molecular Pathology and Immunology, University of Porto, Porto, Portugal

55. Columbia University Mailman School of Public Health, New York, NY, USA

56. Life Sciences Department, Barcelona Supercomputing Center, Barcelona, Spain

57. Department of Clinical Biochemistry and Pharmacology, Ben-Gurion University of the

Negev, Beer-Sheva, Israel

58. National Institute for Biotechnology in the Negev, Beer-Sheva, Israel

59. Leidos Biomedical, Frederick, MD, USA

60. Leidos Biomedical, Rockville, MD, USA

61. UNYTS, Buffalo, NY, USA

62. Washington Regional Transplant Community, Annandale, VA, USA

63. Therapeutics, Roswell Park Comprehensive Cancer Center, Buffalo, NY, USA

64. Gift of Life Donor Program, Philadelphia, PA, USA

65. LifeGift, Houston, TX, USA

66. Center for Organ Recovery and Education, Pittsburgh, PA, USA

67. LifeNet Health, Virginia Beach, VA. USA

68. National Disease Research Interchange, Philadelphia, PA, USA

69. Van Andel Research Institute, Grand Rapids, MI, USA

70. Department of Neurology, University of Miami Miller School of Medicine, Miami, FL, USA

71. Biorepositories and Biospecimen Research Branch, Division of Cancer Treatment and

Diagnosis, National Cancer Institute, Bethesda, MD, USA

72. Temple University, Philadelphia, PA, USA

73. Virgina Commonwealth University, Richmond, VA, USA

74. European Molecular Biology Laboratory, European Bioinformatics Institute, Hinxton, United

Kingdom

75. Genomics Institute, UC Santa Cruz, Santa Cruz, CA, USA

76. Carl Icahn Laboratory, Princeton University, Princeton, NJ, USA

77. Department of Population Health Sciences, The University of Utah, Salt Lake City, Utah, $40 \quad$ USA

78. Schools of Medicine, Engineering, and Public Health, Johns Hopkins University, Baltimore, MD, USA

79. Department of Biostatistics, Bloomberg School of Public Health, Johns Hopkins University, Baltimore, MD, USA

80. Department of Medical Biology, The Walter and Eliza Hall Institute of Medical Research,

Parkville, Victoria, Australia 
81. Altius Institute for Biomedical Sciences, Seattle, WA, USA

82. Division of Genetics, University of Washington, Seattle, WA, University of Washington, Seattle, WA, USA

83. Department of Cardiology, University of Washington, Seattle, WA, USA

84. HudsonAlpha Institute for Biotechnology, Huntsville, AL, USA

85. Genome Sciences, University of Washington, Seattle, WA, USA

86. National Institute of Dental and Craniofacial Research, Bethesda, MD, USA

87. Division of Neuroscience and Basic Behavioral Science, National Institute of Mental Health, National Institutes of Health, Bethesda, MD, USA

88. National Institute on Drug Abuse, Bethesda, MD, USA

89. Office of Strategic Coordination, Division of Program Coordination, Planning and Strategic Initiatives, Office of the Director, National Institutes of Health, Rockville, MD, USA

90. Division of Genomic Medicine, National Human Genome Research Institute, Bethesda, MD, USA

\section{Funding}

The consortium was funded by GTEx program grants: HHSN268201000029C (F.A., K.G.A., A.V.S., X.Li., E.T., S.G., A.G., S.A., K.H.H., D.Y.N., K.H., S.R.M., J.L.N.), 5U41HG009494 (F.A., K.G.A.), 10XS170 (Subcontract to Leidos Biomedical) (W.F.L., J.A.T., G.K., A.M., S.S., R.H., G.Wa., M.J., M.Wa., L.E.B., C.J., J.W., B.R., M.Hu., K.M., L.A.S., H.M.G., M.Mo., L.K.B.), $10 X S 171$ (Subcontract to Leidos Biomedical) (B.A.F., M.T.M., E.K., B.M.G., K.D.R., J.B.), 10ST1035 (Subcontract to Leidos Biomedical) (S.D.J., D.C.R., D.R.V.), R01DA00622717 (D.C.M., D.A.D.), Supplement to University of Miami grant DA006227. (D.C.M., D.A.D.), HHSN261200800001E (A.M.S., D.E.T., N.V.R., J.A.M., L.S., M.E.B., L.Q., T.K., D.B., K.R., A.U.), R01MH101814 (M.M-A., V.W., S.B.M., R.G., E.T.D., D.G-M., A.V.), U01HG007593 (S.B.M.), R01MH101822 (C.D.B.), U01HG007598 (M.O., B.E.S.).

\section{COI}

F.A. is an inventor on a patent application related to TensorQTL; S.E.C. is a co-founder, chief technology officer and stock owner at Variant Bio; E.R.G. is on the Editorial Board of Circulation Research, and does consulting for the City of Hope / Beckman Research Institut; E.T.D. is chairman and member of the board of Hybridstat LTD.; B.E.E. is on the scientific advisory boards of Celsius Therapeutics and Freenome; G.G. receives research funds from IBM and Pharmacyclics, and is an inventor on patent applications related to MuTect, ABSOLUTE, MutSig, POLYSOLVER and TensorQTL; S.B.M. is on the scientific advisory board of Prime Genomics Inc.; D.G.M. is a co-founder with equity in Goldfinch Bio, and has received research support from AbbVie, Astellas, Biogen, BioMarin, Eisai, Merck, Pfizer, and Sanofi-Genzyme; H.K.I. has received speaker honoraria from GSK and AbbVie.; T.L. is a scientific advisory board member of Variant Bio with equity and Goldfinch Bio. P.F. is member of the scientific advisory boards of Fabric Genomics, Inc., and Eagle Genomes, Ltd. P.G.F. is a partner of Bioinf2Bio. 